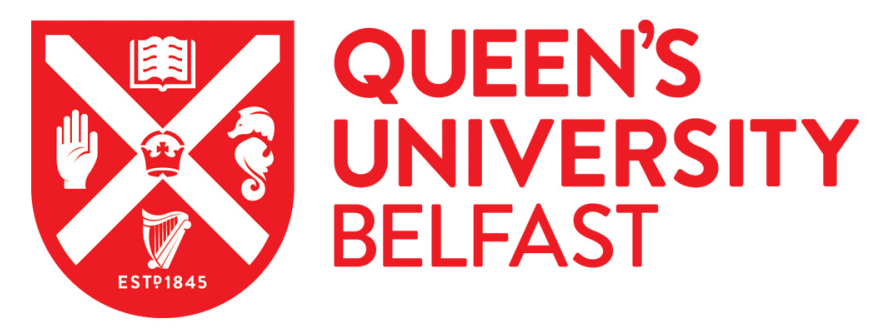

\title{
Power Allocation for Multi-Way Massive MIMO Relaying
}

Chung, H. D., Ngo, H. Q., Matthaiou, M., \& Nguyen, L. D. (2018). Power Allocation for Multi-Way Massive MIMO Relaying. IEEE Transactions on Communications, 66(10), 4457-4472.

https://doi.org/10.1109/TCOMM.2018.2839608

\section{Published in:}

IEEE Transactions on Communications

\section{Document Version:}

Peer reviewed version

\section{Queen's University Belfast - Research Portal:}

Link to publication record in Queen's University Belfast Research Portal

\section{Publisher rights}

(c) 2018 IEEE.

This work is made available online in accordance with the publisher's policies. Please refer to any applicable terms of use of the publisher.

\section{General rights}

Copyright for the publications made accessible via the Queen's University Belfast Research Portal is retained by the author(s) and / or other copyright owners and it is a condition of accessing these publications that users recognise and abide by the legal requirements associated with these rights.

Take down policy

The Research Portal is Queen's institutional repository that provides access to Queen's research output. Every effort has been made to ensure that content in the Research Portal does not infringe any person's rights, or applicable UK laws. If you discover content in the Research Portal that you believe breaches copyright or violates any law, please contact openaccess@qub.ac.uk. 


\title{
Power Allocation for Multi-Way Massive MIMO Relaying
}

\author{
Chung Duc Ho, Student Member, IEEE, Hien Quoc Ngo, Member, IEEE, Michail Matthaiou, Senior Member, \\ IEEE, and Long D. Nguyen, Student Member, IEEE
}

\begin{abstract}
We consider a multi-way decode-and-forward (DF) relaying network with very large antenna arrays at the relay station. In this system, each user and the relay operate in halfduplex and time-division duplexing (TDD) modes. To exchange information among all users, we propose a new transmission protocol which combines massive multiple-input multiple-output (MIMO) technology with linear processing, self-interference cancelation, and successive cancelation decoding. Our proposed transmission protocol reduces the number of time-slots for data exchange among users by approximately 2 times, compared to the conventional data transmission protocol. For this new topology, we derive a very tight approximation of the spectral efficiency in closed-form assuming perfect channel state information (CSI). Then, CSI acquisition method at the relay and the users is provided and analysed. We show via numerical simulations, the performance gap between imperfect and perfect CSI cases is small. The closed-form expression of the spectral efficiency enables us to design two power allocation schemes. In the first power allocation scheme, we choose the transmit powers at the users and the relay to maximize the sum spectral efficiency, subject to a given quality-of-service requirement for each user. In the second power allocation scheme, the objective is the energy efficiency taking into account the hardware power consumption. Both power allocation schemes can be efficiently executed by iteratively solving a sequence of convex problems. Numerical results verify the effectiveness of the proposed transmission protocol and the power allocation schemes compared to the stateof-the-art.
\end{abstract}

Index Terms-Amplify-and-forward, decode-and-forward, maximum-ratio processing, multi-way relay massive MIMO, power allocation, successive cancelation decoding.

\section{INTRODUCTION}

Multi-way relaying has become one of the most promising technologies for next generation wireless systems, for its ability to reliably exchange information among many users and to achieve very high sum spectral efficiency [2]-[4]. As mentioned in [3], many users located in geographically separated locations can exchange their data by using one or several sharing relay networks at the same time-frequency resource. The relay nodes are used to reduce the effect of path loss, and hence, many users can communicate with each other in large regions. In [2], the authors showed that multi-way relaying networks offer a huge spectral efficiency by using linear processing at the relay.

In recent years, massive multiple-input multiple-output (MIMO) technology has been extensively investigated to scaleup the system throughput [5]-[8]. In massive MIMO, many

Manuscript received November 29, 2017; accepted May 15, 2018. The authors are the Institute of Electronics, Communications and Information Technology (ECIT), Queen's University Belfast, BT3 9DT, Belfast, U.K, (email: \{choduc01, hien.ngo, m.matthaiou, lnguyen04\}@qub.ac.uk). This work was supported by project no. 3811/QD-UBND, Binh Duong government, Vietnam. The work of M. Matthaiou was supported in part by the EPSRC under grant EP/P000673/1. Part of this work will appear at the IEEE International Conference on Communications (ICC), May 2018 [1]. users are simultaneously served in the same time-frequency resource by a base station equipped with many antennas. Particularly, in [5]-[7], the authors showed that by using a very large antenna array at the base station, the channel vectors between the users and base station antenna array become pairwisely (nearly) orthogonal. Therefore, with linear processing at the base station, the resulting inter-user interference and noise are negligible, compared to the desired signal. More precisely, by utilizing simple coherent linear processing, such as zero-forcing $(\mathrm{ZF})$ or maximum-ratio (MR) processing, we can steer the desired signals towards the target users in the downlink, and project the undesired signals onto the their orthogonal space to reduce their effects [9], [10]. Thus, the spectral and energy efficiencies of massive MIMO systems can increase ten or even hundred times compared to that of conventional multi-user MIMO systems without exacerbating the system complexity [5], [8]. As a consequence, massive MIMO is considered as a key technology for 5th-generation (5G) wireless communication systems [7], [11].

The combination between multi-way relay networks and massive MIMO technology, known as multi-way massive MIMO relaying, has attracted a significant amount of research interest very recently, since it offers substantial system performance gains in terms of spectral and energy efficiency, and transmit power reductions [12]-[16]. The authors in [12], [13] demonstrated that by exploiting the advantages of massive antennas at the relay, the transmit power utilized by each user and the relay can be reduced proportionally to the number of antennas, while maintaining a given quality-of-service. In [14], the authors derived the asymptotic signal-to-inference-plusnoise ratio (SINR) of amplify-and-forward (AF) muti-way relay networks with very large antenna arrays by assuming perfect channel state information (CSI). The extension of this study was reported in [15] which applied two transmission strategies, namely pairwise and non-pairwise ZF schemes to provide a high SINR and the achievable sum rate of the system. The recent works [16] extended the results of [14], [15] to multi-cell scenario setups. The effects of imperfect CSI and pilot contamination in multi-way relay networks massive MIMO were also assessed. All aforementioned works, however, used the conventional transmission protocol which requires in total $K$ time-slots for information exchange among $K$ users.

Motivated by the above discussion, this paper considers a multi-way decode-and-forward (DF) relay network with massive antenna arrays at the relay station. We aim at designing a new transmission protocol which requires a lower number of time-slots, and hence, increases substantially the spectral efficiency. The main contributions of this paper are summarized as follows:

- We propose a new transmission protocol which relies on massive MIMO technology and successive cance- 
lation decoding. Our analysis showcases that by using our proposed scheme, the number of time-slots for data exchange among users is significantly scaled down (i.e., approximately 2 times), compared to that for the conventional data transmission protocol. As a result, compared to the conventional scheme, our proposed scheme provides a double sum spectral efficiency, when the number of antennas at the relay grows large.

- We derive an analytical and insightful approximation of the spectral efficiency. This approximation is very tight, especially when the number of antennas is large.

- To improve the sum spectral and the energy efficiency, we design two novel power allocation algorithms: sum spectral efficiency maximization and energy efficiency maximization, taking into account the hardware consumption. Our numerical results show that the proposed power allocations offer much better sum spectral and energy efficiency compared to the standard uniform power allocation.

Notations: Upper and lower case boldface letters are used to denote matrices and vectors, respectively. The superscript $(\cdot)^{H}$ represents the conjugate transpose. The notations $\mathbb{E}\{\cdot\}$ and $\operatorname{Tr}(\cdot)$ stand for the expectation and trace operators, respectively. The symbol $\|\cdot\|$ represents the norm of a vector, and $\lceil\cdot\rceil$ represents the ceiling function. We use $\mathbf{z}_{k}$ to denote the $k$-th column of matrix $\mathbf{Z}$, and $\mathbf{I}_{K}$ to denote $K \times K$ identity matrix. Finally, we use the notations $\stackrel{a . s .}{\rightarrow}$ and $\mathbf{Z} \circ \mathbf{Y}$ to denote the almost sure convergence and the Hadamard product of matrices $\mathbf{Z}$ and $\mathbf{Y}$, respectively.

\section{SYSTEM MODEL}

We consider a multi-way DF massive MIMO relaying system, where $K$ single-antenna users exchange their bearingdata among them via the assistance of a common relay station in the same time-frequency resource. The relay station is equipped with a massive antenna array with $M$ antennas. We assume that each user and relay operate in half-duplex mode, and perfect channel state information (CSI) is available at the users and the relay station. ${ }^{1}$ We further assume that the direct links (user-to-user links) do not exist due to large path loss and/or heavy shadowing.

\section{A. Propagation Channel Model}

The propagation channels experience both large-scale fading (including path loss and shadowing) and small-scale fading (here we assume Rayleigh fading). Let $g_{m k}$ be the channel coefficient between the $k$-th user and the $m$-th antenna at the relay. Then,

$$
g_{m k}=\sqrt{\beta_{k}} h_{m k}, m=1, \ldots, M ; k=1, \ldots, K,
$$

where $h_{m k} \sim \mathcal{C N}(0,1)$ represents the small-scale fading, and $\beta_{k}$ models the large-scale fading, which does not depend on

\footnotetext{
${ }^{1}$ The CSI at the relay station can be acquired through uplink pilots under TDD operation [6], while the CSI at the users can be acquired by using the beamforming training scheme of [17]. We assume perfect CSI during our theoretical development since: i) it simplifies the analysis and enables us to get important insights and to further optimize the system performance; and ii) the results obtained under this assumption can be regarded as the upper bounds of what we can achieve with imperfect CSI. Furthermore, in some scenarios, the assumption of perfect CSI is reasonable. For example, in low mobility environments, the coherence interval is large, and hence, we can assign a long duration for training. As a result, the channel estimate is very accurate. The details are provided in Section VII.
}

$m \in\{1,2, \ldots, M\}$ as the distance between the $k$-th user and the relay are much larger than the antenna spacing. Furthermore, $\beta_{k}$ is assumed to be constant over many coherence time intervals and known a priori as it changes very slowly with time. In matrix form, we have

$$
\mathbf{G}=\mathbf{H D}^{1 / 2}
$$

where $\mathbf{G} \in \mathbb{C}^{M \times K}$ is the channel matrix from the $K$ users to $M$ antennas at the relay, $\mathbf{H} \in \mathbb{C}^{M \times K}$ is the small-scale fading matrix, and $\mathbf{D} \in \mathbb{C}^{K \times K}$ is the large-scale fading matrix which is a diagonal matrix with $[\mathbf{D}]_{k k}=\beta_{k}$. The $(m, k)$-th elements of $\mathbf{G}$ and $\mathbf{H}$ are $g_{m k}$ and $h_{m k}$, respectively.

\section{B. Transmission Protocol}

In this multi-way system, each user wants to get all information transmitted from the other $K-1$ users. The transmission protocol consists of two phases: multiple-access (MA) phase and broadcast (BC) phase. In the MA phase, all $K$ users sharing the same frequency band send simultaneously their data to the relay station. Then, the relay station uses a linear decoding technique to detect all signals from all users. In the $\mathrm{BC}$ phase, these decoded signals are processed through a linear precoding technique and broadcasted to all $K$ users.

In the next sections, we will discuss thoroughly the conventional data transmission protocol and our proposed transmission protocol.

\section{CONVENTIONAL TRANSMISSION PROTOCOL}

In this section, firstly, the conventional data transmission scheme for the multi-way DF massive MIMO relaying networks is summarized. Then, closed-form expressions of the corresponding spectral efficiencies are derived. We recall that with the conventional transmission protocol, $K$ time-slots are required for the information exchange among the $K$ users.

\section{A. Multiple-Access Phase}

During this phase, all $K$ users transmit their signals in only one time-slot to the relay station. Let $x_{k}$, where $\mathbb{E}\left\{\left|x_{k}\right|^{2}\right\}=1$, be the signal transmitted from user $k$. Then, the $M \times 1$ received signal vector at the relay can be written as

$$
\mathbf{y}_{\mathrm{R}}=\sum_{k=1}^{K} \sqrt{P_{\mathrm{u}, k}} \mathbf{g}_{k} x_{k}+\mathbf{n}_{\mathrm{R}}
$$

where $P_{\mathrm{u}, k}$ is the transmit power of the $k$-th user (normalized over the noise power), $\mathbf{g}_{k}$ is the $k$-th column of the channel matrix $\mathbf{G}$, and $\mathbf{n}_{\mathrm{R}} \in \mathbb{C}^{M \times 1}$ is the noise vector with independent and identically distributed (i.i.d.) $\mathcal{C N}(0,1)$ elements.

From the received signal vector given in (3), the relay station will detect all $K$ signals $x_{k}, k=1, \ldots, K$. We assume that the relay station uses maximum-ratio combining scheme to detect $x_{k}$. Maximum-ratio combing scheme is used since it is simple and can be implemented in a distributed manner. Furthermore, in this work we focus on the use of massive antenna arrays at the relay station, and hence, maximumratio combining technique works very well [11]. With the maximum-ratio combing scheme, the received signal vector $\mathbf{y}_{\mathrm{R}}$ is first multiplied with the channel $\mathbf{G}^{H}$ as

$$
\mathbf{r}=\mathbf{G}^{H} \mathbf{y}_{\mathrm{R}}=\mathbf{G}^{H} \sum_{k=1}^{K} \sqrt{P_{\mathrm{u}, k}} \mathbf{g}_{k} x_{k}+\mathbf{G}^{H} \mathbf{n}_{\mathrm{R}} .
$$


Then $x_{k}$ will be detected from the $k$-th element of $\mathbf{r}$, denoted by $r_{k}$. From (4), $r_{k}$ can be written as

$$
r_{k}=\sqrt{P_{\mathrm{u}, k}}\left\|\mathbf{g}_{k}\right\|^{2} x_{k}+\sum_{i=1, i \neq k}^{K} \sqrt{P_{\mathrm{u}, i}} \mathbf{g}_{k}^{H} \mathbf{g}_{i} x_{i}+\mathbf{g}_{k}^{H} \mathbf{n}_{\mathrm{R}} .
$$

Thus, the uplink spectral efficiency (measured in bit/s/Hz) of the $k$-th user is

$$
\mathrm{R}_{k}^{\mathrm{ul}}=\mathbb{E}\left\{\log _{2}\left(1+\frac{P_{\mathrm{u}, k}\left\|\mathbf{g}_{k}\right\|^{4}}{\sum_{i=1, i \neq k}^{K} P_{\mathrm{u}, i}\left|\mathbf{g}_{k}^{H} \mathbf{g}_{i}\right|^{2}+\left\|\mathbf{g}_{k}\right\|^{2}}\right)\right\}
$$

Similar to [18], we can use Jensen's inequality to obtain a rigorous lower bound of the spectral efficiency (6) in a simple closed-form expression as:

$$
\begin{aligned}
\mathrm{R}_{k}^{\mathrm{ul}} \geq \tilde{\mathrm{R}}_{k}^{\mathrm{ul}} & =\log _{2}\left(1+\mathbb{E}\left\{\frac{\sum_{\substack{i=1 \\
i \neq k}}^{K} P_{\mathrm{u}, i}\left|\mathbf{g}_{k}^{H} \mathbf{g}_{i}\right|^{2}+\left\|\mathbf{g}_{k}\right\|^{2}}{P_{\mathrm{u}, k}\left\|\mathbf{g}_{k}\right\|^{4}}\right\}^{-1}\right) \\
& =\log _{2}\left(1+\frac{P_{\mathrm{u}, k}(M-1) \beta_{k}}{\sum_{i=1, i \neq k}^{K} P_{\mathrm{u}, i} \beta_{i}+1}\right) .
\end{aligned}
$$

\section{B. Broadcast Phase}

In this phase, in order to broadcast all signals (which are decoded in the MA phase) to all $K$ users, the relay station spends $K-1$ time-slots. In the $t$-th time-slot, the relay want to send $x_{j(k, t)}$ to the $k$-th user, $k=1, \ldots, K$, where

$$
j(k, t) \triangleq\left\{\begin{array}{cc}
(k+t) \text { modulo } K, & \text { if } \begin{array}{c}
(k+t) \neq K \\
K,
\end{array} \\
\text { otherwise. }
\end{array}\right.
$$

We assume that the relay uses maximum-ratio transmission technique to process signals in each time-slot before beamforming them to all $K$ users. Therefore, the transmitted signal vector at the relay in the $t$-th time-slot is

$$
\mathbf{s}^{(t)}=\sum_{i=1}^{K} \sqrt{\eta_{j(i, t)}} \mathbf{g}_{i} x_{j(i, t)},
$$

where $\left\{\eta_{j(i, t)}\right\}, i=1, \ldots, K$, are the power control coefficients which are chosen to satisfy a given power constraint at the relay:

$$
\mathbb{E}\left\{\left\|\mathbf{s}^{(t)}\right\|^{2}\right\} \leqslant P_{\mathrm{r}, \mathrm{th}} .
$$

From (9), (10), and the identity $\mathbb{E}\left\{\left\|\mathbf{g}_{i}\right\|^{2}\right\}=M \beta_{i}$, we obtain the constraint

$$
\sum_{i=1}^{K} \eta_{j(i, t)} \beta_{i} \leqslant \frac{P_{\mathrm{r}, \mathrm{th}}}{M}
$$

With the transmitted signal vector (9), the $k$-th user sees

$y_{k}^{(t)}=\mathbf{g}_{k}^{H} \mathbf{s}^{(t)}+n_{k}^{(t)}=\sum_{i=1}^{K} \sqrt{\eta_{j(i, t)}} \mathbf{g}_{k}^{H} \mathbf{g}_{i} x_{j(i, t)}+n_{k}^{(t)}$.

The $k$-th user is able to eliminate its self-interference before decoding its desired signal $x_{j(k, t)}$, as it perfectly knows its own transmitted signal $x_{k}$ (or $\left.x_{j(k-t, t)}\right)$. Therefore, after selfinterference cancelation, the received signal is given by

$$
\begin{aligned}
\tilde{y}_{k}^{(t)} & =\sqrt{\eta_{j(k, t)}}\left\|\mathbf{g}_{k}\right\|^{2} x_{j(k, t)} \\
& +\sum_{\substack{i=1 \\
j(i, t) \neq j(k, t), j(k-t, t)}}^{K} \sqrt{\eta_{j(i, t)}} \mathbf{g}_{k}^{H} \mathbf{g}_{i} x_{j(i, t)}+n_{k}^{(t)} .
\end{aligned}
$$

Therefore, the corresponding downlink spectral efficiency of the $k$-th user in the $t$-th time-slot is

$$
\mathrm{R}_{k}^{\mathrm{dl},(t)}=\mathbb{E}\left\{\log _{2}\left(1+\frac{\eta_{j(k, t)} \mid \mathbf{g}_{k} \|^{4}}{\sum_{\substack{i=1 \\ j(i, t) \neq j(k, t), j(k-t, t)}}^{K} \eta_{j(i, t)}\left|\mathbf{g}_{k}^{H} \mathbf{g}_{i}\right|^{2}+1}\right)\right\} .
$$

By using Jensen's inequality, we obtain a simple closedform result for a tight lower bound of $\mathrm{R}_{k}^{\mathrm{dl},(t)}$ as in the following proposition.

Proposition 1: The spectral efficiency $\mathrm{R}_{k}^{\mathrm{dl},(t)}$ given by (14) can be lower bounded by

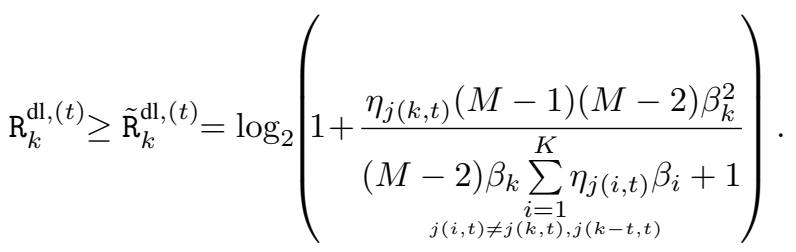

Proof: By following the same methodology as in [18, Appendix A], and using [19, Lemma 2.10], we can arrive at (15).

\section{Multi-Way Transmission with Successive CANCELATION DECODING}

In this section, a novel data transmission protocol is proposed via the use of the successive cancelation decoding principle. The most significant improvement of this new protocol is that only $\left\lceil\frac{K-1}{2}\right\rceil+1$ time-slots are required. Note that, perfect cancelation of interference is not our objective. Instead, to ensure that aggregated interference, including selfinterference and inter-user interference, does not deteriorate the system performance, we apply the properties of massive MIMO together with successive self-interference cancelation, and ZF decoding at the users. Therefore, when the number of antennas $M$ goes to infinity, inter-user interference can be minimized significantly.

\section{A. Multiple-Access Phase}

This phase requires one time-slot and is the same as that of the conventional transmission protocol. See Section III-A.

\section{B. Broadcast Phase}

Instead of spending $K-1$ time-slots to broadcast all signals to all $K$ users as in the conventional scheme, here we propose to use only $\left\lceil\frac{K-1}{2}\right\rceil$ time-slots. The key idea of this method is that: at a given time-slot, the $k$-th user subtracts all interference sources caused by signals decoded in previous time-slots prior to decoding the desired signal. In addition, after $\left\lceil\frac{K-1}{2}\right\rceil$ timeslots and with successive interference cancellation, the $k$ th user receives $\left\lceil\frac{K-1}{2}\right\rceil$ signals, and each signal is a linear 
combination of $K-\left\lceil\frac{K-1}{2}\right\rceil-1$ symbols. Since $\left\lceil\frac{K-1}{2}\right\rceil \geq$ $K-\left\lceil\frac{K-1}{2}\right\rceil-1$, the $k$-th user can use the zero-forcing technique to decode all $K-\left\lceil\frac{K-1}{2}\right\rceil-1$ symbols without any inter-user interference. A detailed discussion of the proposed scheme is now presented.

1) First time-slot: The relay wants to send $x_{j(k, 1)}$ to the $k$ th user, for $k=1, \ldots, K$. To do this, it forms a signal vector to broadcast to the users as:

$$
\mathbf{s}^{(1)}=\sum_{i=1}^{K} \sqrt{\eta_{j(i, 1)}^{(1)}} \mathbf{g}_{i} x_{j(i, 1)},
$$

where $\left\{\eta_{j(i, 1)}^{(1)}\right\}, i=1, \ldots, K$, are the power control coefficients at the relay in the first time-slot which are chosen to satisfy a given power constraint at the relay $\mathbb{E}\left\{\left\|\mathbf{s}^{(1)}\right\|^{2}\right\} \leqslant P_{\mathrm{r}, \mathrm{th}}$, or

$$
M \sum_{i=1}^{K} \eta_{j(i, 1)}^{(1)} \beta_{i} \leqslant P_{\mathrm{r}, \mathrm{th}} .
$$

Then, the $k$-th user receives

$$
\begin{aligned}
y_{k}^{(1)} & =\mathbf{g}_{k}^{H} \mathbf{s}^{(1)}+n_{k}^{(1)} \\
& =\sum_{i=1}^{K} \sqrt{\eta_{j(i, 1)}^{(1)}} \mathbf{g}_{k}^{H} \mathbf{g}_{i} x_{j(i, 1)}+n_{k}^{(1)},
\end{aligned}
$$

where $n_{k}^{(1)} \sim \mathcal{C N}(0,1)$ is the additive noise at the $k$-th user in the first time-slot. Before detecting the desired signal $x_{j(k, 1)}$, the $k$-th user performs self-interference cancelation by subtracting its transmitted signal $x_{k}\left(\right.$ or $\left.x_{j(k-1,1)}\right)$ from $y_{k}^{(1)}$. After self-interference cancelation, the received signal at the $k$-th user becomes

$$
\begin{aligned}
\tilde{y}_{k}^{(1)} & =\sqrt{\eta_{j(k, 1)}^{(1)}}\left\|\mathbf{g}_{k}\right\|^{2} x_{j(k, 1)} \\
& +\sum_{\substack{i=1 \\
j(i, 1) \notin \mathcal{V}_{k, 1}}}^{K} \sqrt{\eta_{j(i, 1)}^{(1)}} \mathbf{g}_{k}^{H} \mathbf{g}_{i} x_{j(i, 1)}+n_{k}^{(1)},
\end{aligned}
$$

where

$$
\mathcal{V}_{k, t} \triangleq\{j(k-t, t), j(k-t+1, t), \ldots, j(k, t)\} .
$$

The first term of (19) represents the desired signal, the second and third terms are interference plus noise, respectively. Thus, we obtain the corresponding spectral efficiency as

$$
\mathrm{R}_{k}^{\mathrm{dl},(1)}=\mathbb{E}\left\{\log _{2}\left(1+\frac{\eta_{j(k, 1)}^{(1)}\left\|\mathbf{g}_{k}\right\|^{4}}{\sum_{\substack{i=1 \\ j(i, 1) \notin \mathcal{V}_{k, 1}}}^{K} \eta_{j(i, 1)}^{(1)}\left|\mathbf{g}_{k}^{H} \mathbf{g}_{i}\right|^{2}+1}\right)\right\}
$$

2) Second time-slot: After aiming to transmit $x_{j(k, 1)}$ to the $k$-th user in the fist time-slot, the relay next wants to send $x_{j(k, 2)}$ to the $k$-th user, $k=1, \ldots, K$. So, it precodes the transmitted signals as

$$
\mathbf{s}^{(2)}=\sum_{i=1}^{K} \sqrt{\eta_{j(i, 2)}^{(2)}} \mathbf{g}_{i} x_{j(i, 2)},
$$

where $\left\{\eta_{j(i, 2)}^{(2)}\right\}, i=1, \ldots, K$, are the power control coefficients at the relay in the second time-slot chosen to satisfy a given power constraint $P_{\mathrm{r}, \text { th }}$ at the relay as

$$
M \sum_{i=1}^{K} \eta_{j(i, 2)}^{(2)} \beta_{i} \leqslant P_{\mathrm{r}, \mathrm{th}}
$$

Then, the $k$-th user receives

$$
\begin{aligned}
y_{k}^{(2)} & =\mathbf{g}_{k}^{H} \mathbf{s}^{(2)}+n_{k}^{(2)} \\
& =\sum_{i=1}^{K} \sqrt{\eta_{j(i, 2)}^{(2)}} \mathbf{g}_{k}^{H} \mathbf{g}_{i} x_{j(i, 2)}+n_{k}^{(2)} .
\end{aligned}
$$

Since user $k$ knows its own transmitted signal $x_{k}$ (or $x_{j(k-1,1)}$ ) and the symbol detected in the first time-slot $x_{j(k, 1)}$, it can perform interference cancelation by removing these symbols from $y_{k}^{(2)}$ before detecting the desired signal $x_{j(k, 2)}$. After interference cancelation, the received signal at the $k$-th user becomes

$$
\begin{aligned}
\tilde{y}_{k}^{(2)} & =\sqrt{\eta_{j(k, 2)}^{(2)}}\left\|\mathbf{g}_{k}\right\|^{2} x_{j(k, 2)} \\
& +\sum_{\substack{i=1 \\
j(i, 2) \notin \mathcal{V}}}^{K} \sqrt{\eta_{j(i, 2)}^{(2)}} \mathbf{g}_{k}^{H} \mathbf{g}_{i} x_{j(i, 2)}+n_{k}^{(2)} .
\end{aligned}
$$

The corresponding spectral efficiency of the $k$-th user in the broadcast phase at the second time-slot is given by

$$
\mathrm{R}_{k}^{\mathrm{dl},(2)}=\mathbb{E}\left\{\log _{2}\left(1+\frac{\eta_{j(k, 2)}^{(2)}\left\|\mathbf{g}_{k}\right\|^{4}}{\sum_{\substack{i=1 \\ j(i, 2) \notin \mathcal{V} \\ k, 2}}^{K} \eta_{j(i, 2)}^{(2)}\left|\mathbf{g}_{k}^{H} \mathbf{g}_{i}\right|^{2}+1}\right)\right\} .
$$

3) $t$-th time-slot: At the $t$-th time-slot, the relay intends to send $x_{j(k, t)}$ to the $k$-th user, for $k=1, \ldots, K$. The signal vector transmitted from the relay is

$$
\mathbf{s}^{(t)}=\sum_{i=1}^{K} \sqrt{\eta_{j(i, t)}^{(t)}} \mathbf{g}_{i} x_{j(i, t)} .
$$

As before, $\left\{\eta_{j(i, t)}^{(t)}\right\}, i=1, \ldots, K$, are the power control coefficients at the $t$-th time-slot chosen to satisfy

$$
M \sum_{i=1}^{K} \eta_{j(i, t)}^{(t)} \beta_{i} \leqslant P_{\mathrm{r}, \mathrm{th}} .
$$

Then, the signal received at the $k$-th user is

$$
\begin{aligned}
y_{k}^{(t)} & =\mathbf{g}_{k}^{H} \mathbf{s}^{(t)}+n_{k}^{(t)} \\
& =\sum_{i=1}^{K} \sqrt{\eta_{j(i, t)}^{(t)}} \mathbf{g}_{k}^{H} \mathbf{g}_{i} x_{j(i, t)}+n_{k}^{(t)},
\end{aligned}
$$

where $n_{k}^{(1)} \sim \mathcal{C N}(0,1)$ denotes the additive noise. At the $t$-th time-slot, the $k$-th user detected $\left\{x_{j(k, 1)}, x_{j(k, 2)}\right.$,

$\left.\ldots, x_{j(k, t-1)}\right\}$ in previous time-slots. In addition, it knows it own transmitted signal $x_{j(k-1,1)}$. So, user $k$ can remove these 
symbols from $y_{k}^{(t)}$ before detecting $x_{j(k, t)}$. The received signal at the $k$-th user after interference cancelation is

$$
\begin{aligned}
\tilde{y}_{k}^{(t)} & =\sqrt{\eta_{j(k, t)}^{(t)}}\left\|\mathbf{g}_{k}\right\|^{2} x_{j(k, t)} \\
& +\sum_{\substack{i=1 \\
j(i, t) \notin \mathcal{V}_{k, t}}}^{K} \sqrt{\eta_{j(i, t)}^{(t)}} \mathbf{g}_{k}^{H} \mathbf{g}_{i} x_{j(i, t)}+n_{k}^{(t)} .
\end{aligned}
$$

Therefore, the spectral efficiency of the $k$-th user at the $t$-th time-slot in the broadcast phase is given by

$$
\mathrm{R}_{k}^{\mathrm{dl},(t)}=\mathbb{E}\left\{\log _{2}\left(1+\frac{\eta_{j(k, t)}^{(t)}\left\|\mathbf{g}_{k}\right\|^{4}}{\sum_{\substack{i=1 \\ j(i, t) \notin \mathcal{V} \\ k, t}}^{K} \eta_{j(i, t)}^{(t)}\left|\mathbf{g}_{k}^{H} \mathbf{g}_{i}\right|^{2}+1}\right)\right\} .
$$

By following a similar methodology as in the derivation of the spectral efficieny in Proposition 1, we obtain a tight lower bound on the spectral efficiency (31) as in Proposition 2.

Proposition 2: The spectral efficiency $\mathrm{R}_{k}^{\mathrm{dl},(t)}$ given by (31) can be lower bounded by

$$
\mathrm{R}_{k}^{\mathrm{dl},(t)} \geq \tilde{\mathrm{R}}_{k}^{\mathrm{dl},(t)}=\log _{2}\left(1+\frac{\eta_{j(k, t)}^{(t)}(M-1)(M-2) \beta_{k}^{2}}{(M-2) \beta_{k} \sum_{\substack{i=1 \\ j(i, t) \notin \mathcal{V} \\ k, t}}^{K} \eta_{j(i, t)}^{(t)} \beta_{i}+1}\right) .
$$

4) After $t^{\prime}=\left\lceil\frac{K-1}{2}\right\rceil$ time-slots, the $k$-th user has received $t^{\prime}$ signals $\left\{y_{k}^{(1)}, \ldots, y_{k}^{\left(t^{\prime}\right)}\right\}$, where $y_{k}^{(t)}$ is given in (29) for $t=1, \ldots, t^{\prime}$. In addition, it has decoded $t^{\prime}$ symbols $\left\{x_{j(k, 1)}, \ldots, x_{j\left(k, t^{\prime}\right)}\right\}$. Thus, the $k$-user can perform interference cancelation by subtracting all $t^{\prime}$ detected symbols as well as it own transmitted symbol from each received signal, and obtain the following results:

$$
\left\{\begin{array}{l}
\bar{y}_{k, 1}^{\left(t^{\prime}\right)}=\sum_{\substack{i=1 \\
j\left(i, t^{\prime}\right) \notin \mathcal{V} \\
k, t^{\prime}}}^{K} \sqrt{\eta_{j\left(i, t^{\prime}\right)}^{\left(t^{\prime}\right)}} \mathbf{g}_{k}^{H} \mathbf{g}_{j(k, i-k)} x_{j\left(i, t^{\prime}\right)}+n_{k, 1}^{\left(t^{\prime}\right)} \\
\bar{y}_{k, 2}^{\left(t^{\prime}\right)}=\sum_{\substack{i=1 \\
j\left(i, t^{\prime}\right) \notin \mathcal{V} \\
k, t^{\prime}}}^{K} \sqrt{\eta_{j\left(i, t^{\prime}\right)}^{\left(t^{\prime}-1\right)}} \mathbf{g}_{k}^{H} \mathbf{g}_{j(k, i-k+1)} x_{j\left(i, t^{\prime}\right)}+n_{k, 2}^{\left(t^{\prime}\right)} \\
\vdots \\
\bar{y}_{k, t^{\prime}}^{\left(t^{\prime}\right)}=\sum_{\substack{i=1 \\
j\left(i, t^{\prime}\right) \notin \mathcal{V}_{k, t^{\prime}}}}^{K} \sqrt{\eta_{j\left(i, t^{\prime}\right)}^{\left(t^{\prime}-\left(t^{\prime}-1\right)\right)}} \mathbf{g}_{k}^{H} \mathbf{g}_{j\left(k, i-k+t^{\prime}-1\right)} x_{j\left(i, t^{\prime}\right)}+n_{k, t^{\prime}}^{\left(t^{\prime}\right)}
\end{array}\right.
$$

where $\bar{y}_{k, t}^{\left(t^{\prime}\right)}$ is obtained from $y_{k}^{(t)}$ after performing interference cancelation, and $n_{k, t^{\prime}}^{\left(t^{\prime}\right)}$ is the corresponding noise at user $k$.

Denote by

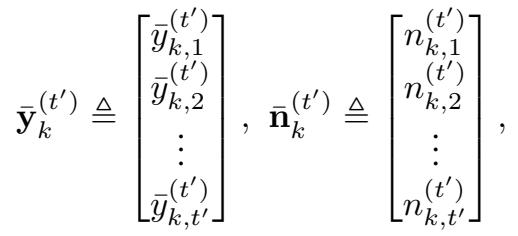

$$
\begin{aligned}
& \overline{\mathbf{x}} \triangleq\left[\begin{array}{llll}
x_{j\left(k, t^{\prime}+1\right)} & x_{j\left(k, t^{\prime}+2\right)} & \ldots & x_{j(k, K-1)}
\end{array}\right]^{T} \text {, }
\end{aligned}
$$

and

$$
\overline{\mathbf{A}}_{k}^{\left(t^{\prime}\right)}=\mathbf{A}_{k}^{\left(t^{\prime}\right)} \circ \mathbf{E}_{\eta}^{\left(t^{\prime}\right)},
$$

where the matrices $\mathbf{A}_{k}^{\left(t^{\prime}\right)} \in \mathbb{C}^{t^{\prime} \times\left(K-t^{\prime}-1\right)}$ and $\mathbf{E}_{k}^{\left(t^{\prime}\right)} \in$ $\mathbb{R}^{t^{\prime} \times\left(K-t^{\prime}-1\right)}$ in (35) are defined as

$\mathbf{A}_{k}^{\left(t^{\prime}\right)} \triangleq\left[\begin{array}{cccc}\mathbf{g}_{k}^{H} \mathbf{g}_{j(k, 1)} & \mathbf{g}_{k}^{H} \mathbf{g}_{j(k, 2)} & \ldots & \mathbf{g}_{k}^{H} \mathbf{g}_{j\left(k, K-t^{\prime}-1\right)} \\ \mathbf{g}_{k}^{H} \mathbf{g}_{j(k, 2)} & \mathbf{g}_{k}^{H} \mathbf{g}_{j(k, 3)} & \ldots & \mathbf{g}_{k}^{H} \mathbf{g}_{j\left(k, K-t^{\prime}\right)} \\ \vdots & \vdots & & \vdots \\ \mathbf{g}_{k}^{H} \mathbf{g}_{j\left(k, t^{\prime}\right)} & \mathbf{g}_{k}^{H} \mathbf{g}_{j\left(k, t^{\prime}+1\right)} & \ldots & \mathbf{g}_{k}^{H} \mathbf{g}_{j(k, K-2)}\end{array}\right]$,

and

$$
\mathbf{E}_{\eta}^{\left(t^{\prime}\right)} \triangleq\left[\begin{array}{cccc}
\sqrt{\eta_{j\left(k, t^{\prime}+1\right)}^{\left(t^{\prime}\right)}} & \sqrt{\eta_{j\left(k, t^{\prime}+2\right)}^{\left(t^{\prime}\right)}} & \cdots & \sqrt{\eta_{j(k, K-1)}^{\left(t^{\prime}\right)}} \\
\sqrt{\eta_{j\left(k, t^{\prime}+1\right)}^{\left(t^{\prime}-1\right)}} & \sqrt{\eta_{j\left(k, t^{\prime}+2\right)}^{\left(t^{\prime}-1\right)}} & \cdots & \sqrt{\eta_{j(k, K-1)}^{\left(t^{\prime}-1\right)}} \\
\vdots & \vdots & & \vdots \\
\sqrt{\eta_{j\left(k, t^{\prime}+1\right)}^{\left(t^{\prime}-\left(t^{\prime}-1\right)\right)}} & \sqrt{\eta_{j\left(k, t^{\prime}+2\right)}^{\left.\left(t^{\prime}-t^{\prime}-1\right)\right)}} & \cdots \sqrt{\eta_{j(k, K-1)}^{\left(t^{\prime}-\left(t^{\prime}-1\right)\right)}}
\end{array}\right] .
$$

Then, (33) can be rewritten in matrix-vector form as

$$
\overline{\mathbf{y}}_{k}^{\left(t^{\prime}\right)}=\overline{\mathbf{A}}_{k}^{\left(t^{\prime}\right)} \overline{\mathbf{x}}+\overline{\mathbf{n}}_{k}^{\left(t^{\prime}\right)}
$$

We can see that $\overline{\mathbf{A}}_{k}^{\left(t^{\prime}\right)}$ is a $t^{\prime} \times\left(K-t^{\prime}-1\right)$ matrix. Since $t^{\prime} \geq\left(K-t^{\prime}-1\right), \overline{\mathbf{A}}_{k}^{\left(t^{\prime}\right)}$ is full column rank, and hence, we can employ the zero-forcing technique to detect all remaining symbols without inter-user interference. With the $\mathrm{ZF}$ technique, $\overline{\mathbf{y}}_{k}^{\left(t^{\prime}\right)}$ is first processed by multiplying it with the pseudo inverse of $\overline{\mathbf{A}}_{k}^{\left(t^{\prime}\right)}$ as

$$
\tilde{\mathbf{r}}_{k}^{\left(t^{\prime}\right)}=\mathbf{Z}^{T} \overline{\mathbf{y}}_{k}^{\left(t^{\prime}\right)}=\mathbf{Z}^{T} \overline{\mathbf{A}}_{k}^{\left(t^{\prime}\right)} \overline{\mathbf{x}}+\mathbf{Z}^{T} \overline{\mathbf{n}}_{k}^{\left(t^{\prime}\right)},
$$

where

$$
\mathbf{Z}^{T} \triangleq\left(\left(\overline{\mathbf{A}}_{k}^{\left(t^{\prime}\right)}\right)^{H} \overline{\mathbf{A}}_{k}^{\left(t^{\prime}\right)}\right)^{-1}\left(\overline{\mathbf{A}}_{k}^{\left(t^{\prime}\right)}\right)^{H} .
$$

Then, $x_{j\left(k, t^{\prime}+n\right)}$ will be detected from the $n$-th element of $\tilde{\mathbf{r}}_{k}^{\left(t^{\prime}\right)}$. Since $\mathbf{Z}^{T} \overline{\mathbf{A}}_{k}^{\left(t^{\prime}\right)}=\mathbf{I}_{K-\left(t^{\prime}+1\right)}$, (39) becomes

$$
\tilde{\mathbf{r}}_{k}^{\left(t^{\prime}\right)}=\overline{\mathbf{x}}+\mathbf{Z}^{T} \overline{\mathbf{n}}_{k}^{\left(t^{\prime}\right)}
$$

and hence, the $n$-th element of $\tilde{\mathbf{r}}_{k}^{\left(t^{\prime}\right)}$ is

$$
\tilde{\mathbf{r}}_{k, n}^{\left(t^{\prime}\right)}=x_{j\left(k, t^{\prime}+n\right)}+\mathbf{z}_{n}^{T} \overline{\mathbf{n}}_{k}^{\left(t^{\prime}\right)} .
$$

Thus, the corresponding spectral efficiency of (42) is

$$
\begin{aligned}
& \mathrm{R}_{k}^{\mathrm{dl},\left(t^{\prime}+n\right)}=\mathbb{E}\left\{\log _{2}\left(1+\frac{1}{\left\|\mathbf{z}_{n}\right\|^{2}}\right)\right\} \\
& =\mathbb{E}\left\{\log _{2}\left(1+\frac{1}{\left[\left(\left(\overline{\mathbf{A}}_{k}^{\left(t^{\prime}\right)}\right)^{H} \overline{\mathbf{A}}_{k}^{\left(t^{\prime}\right)}\right)^{-1}\right]_{n n}}\right)\right\} .
\end{aligned}
$$

We can see that (43) involves a matrix inversion which renders it intractable for further manipulations. It is very difficult to derive an exact closed-form. To allow an analytical derivation, we can approximate the spectral efficiency (43) by using the trace lemma and law of large numbers (as $M$ goes 
to infinity) [20]. The approximating result is presented in the following proposition.

Proposition 3: As $M \rightarrow \infty$, the spectral efficiency $\mathrm{R}_{k}^{\mathrm{dl},\left(t^{\prime}+n\right)}$ given by (43) converges to

$$
\begin{aligned}
& \mathrm{R}_{k}^{\mathrm{dl},\left(t^{\prime}+n\right)} \rightarrow \tilde{\mathrm{R}}_{k}^{\mathrm{dl},\left(t^{\prime}+n\right)} \\
& =\log _{2}\left(1+M \beta_{k} \sum_{i=1}^{t^{\prime}} \eta_{j\left(k, t^{\prime}+n\right)}^{\left(t^{\prime}-i+1\right)} \beta_{j(k, n+i-1)}\right), \\
& n=1, \ldots, K-t^{\prime}-1 .
\end{aligned}
$$

Proof: See Appendix A.

Note that, the conventional transmission scheme does not require successfully decoding of the first $\left\lceil\frac{K-1}{2}\right\rceil$ time-slots. By contrast, our proposed scheme requires successfully decoding of the first $\left[\frac{K-1}{2}\right]$ time-slots to enable the performance of the $\mathrm{ZF}$ technique. However, due to the use of massive antenna arrays at the relay, interference and noise can be cancelled out, and hence, the signal detections in the first $\left[\frac{K-1}{2}\right]$ timeslots are successful with very high probability. Numerical results will show that our proposed scheme outperforms the conventional scheme.

\section{SPECTRAl EFFiCIENCY MaXimizATION}

In this section, we aim at selecting the power coefficients in order to maximize the sum spectral efficiency of our proposed transmission protocol in Section IV under the constraints on per-user spectral efficiency and transmit power at the relay and the users. The sum spectral efficiency is defined as

$$
\tilde{\mathrm{SE}}_{\text {sum }}=\frac{1}{t^{\prime}+1} \sum_{k=1}^{K} \sum_{t=1}^{K-1} \min \left(\tilde{\mathrm{R}}_{j(k, t)}^{\mathrm{ul}}, \tilde{\mathrm{R}}_{k}^{\mathrm{dl},(t)}\right) \text {, }
$$

where $\tilde{\mathrm{R}}_{j(k, t)}^{\mathrm{ul}}$ is given by (7), $\tilde{\mathrm{R}}_{k}^{\mathrm{dl},(t)}$ is given by (32) for $t=$ $1, \ldots, t^{\prime}$ and given by (44) for $t=t^{\prime}+1, \ldots, K-1$. The optimization problem is formulated as follows:

$$
\begin{aligned}
& \underset{P_{\mathrm{u}, k}, \eta_{j(k, t)}^{(t)}}{\operatorname{maximize}} \quad \tilde{\mathrm{SE}}_{\mathrm{sum}} \\
& \text { s.t. } \quad 0 \leqslant P_{\mathrm{u}, k} \leqslant P_{\mathrm{u}, \mathrm{th}}, k=1, \ldots, K \\
& \quad M \sum_{i=1}^{K} \eta_{j(i, t)}^{(t)} \beta_{i} \leqslant P_{\mathrm{r}, \mathrm{th}}, t=1, \ldots, t^{\prime} \\
& \quad \tilde{\mathrm{R}}_{k}^{\mathrm{ul}} \geq \mathrm{R}_{\mathrm{th}, k}^{\mathrm{ul}}, k=1, \ldots, K \\
& \quad \tilde{\mathrm{R}}_{k}^{\mathrm{dl},(t)} \geq \mathrm{R}_{\mathrm{th}, k}^{\mathrm{dl},(t)}, k=1, \ldots, K, t=1, \ldots, K-1,
\end{aligned}
$$

where $P_{\mathrm{u}, \mathrm{th}}, P_{\mathrm{r}, \mathrm{th}}$ are the power constraints of each user and the relay station, $\mathrm{R}_{\mathrm{th}, k}^{\mathrm{ul}}$ and $\mathrm{R}_{\mathrm{th}, k}^{\mathrm{dl},(t)}$ are defined as the threshold uplink and downlink spectral efficiencies.

The above optimization problem is very complicated, and hence, its optimal solution is very difficult to find. To simplify the problem, we propose a sub-optimal solution which first decouples the problem (46) into two optimization problems: i) power allocation optimization across users in the uplink phase, and ii) power allocation optimization at the relay in the downlink phase. Then, these problems will be solved based on the sequential convex approximation (SCA) method [21].

\section{A. Power Allocation Optimization Across Users}

The power allocation optimization problem across users in the uplink phase is formulated as

$$
\begin{aligned}
\underset{P_{\mathrm{u}, k}}{\operatorname{maximize}} & \sum_{k=1}^{K} \sum_{t=1}^{K-1} \tilde{\mathrm{R}}_{j(k, t)}^{\mathrm{ul}} \\
\text { s.t. } & 0 \leqslant P_{\mathrm{u}, k} \leqslant P_{\mathrm{u}, \mathrm{th}}, k=1, \ldots, K \\
& \tilde{\mathrm{R}}_{k}^{\mathrm{ul}} \geq \mathrm{R}_{\mathrm{th}, k}^{\mathrm{ul}}, \quad k=1, \ldots, K .
\end{aligned}
$$

From (7) and the definition of $j(k, t)$ in (8), the optimization problem (47) is equivalent to

$$
\begin{aligned}
& \underset{P_{\mathrm{u}, k}}{\operatorname{maximize}} \sum_{k=1}^{K} \tilde{\mathrm{R}}_{k}^{\mathrm{ul}} \\
& \text { s.t. } \quad 0 \leqslant P_{\mathrm{u}, k} \leqslant P_{\mathrm{u}, \mathrm{th}}, k=1, \ldots, K \\
& \log _{2}\left(1+\frac{P_{\mathrm{u}, k}(M-1) \beta_{k}}{\sum_{\substack{i=1 \\
i \neq k}}^{K} P_{\mathrm{u}, i} \beta_{i}+1}\right) \geq \mathrm{R}_{\mathrm{th}, k}^{\mathrm{ul}}, k=1, \ldots, K,
\end{aligned}
$$

which can be further rewritten as

$$
\left.\begin{array}{l}
\underset{P_{\mathrm{u}, k}}{\operatorname{maximize}} \sum_{k=1}^{K} \frac{1}{\ln 2} \\
\times \quad \ln \left(1+\frac{1}{\frac{1}{P_{\mathrm{u}, k}(M-1) \beta_{k}}\left(\sum_{\substack{i=1 \\
i \neq k}}^{K} P_{\mathrm{u}, i} \beta_{i}+1\right)}\right),
\end{array}\right)
$$

We denote $x=x_{k}=\frac{1}{P_{u, k}(M-1) \beta_{k}}, y=y_{k}=$ $\sum_{i=1, i \neq k}^{K} P_{\mathrm{u}, i} \beta_{i}+1$, and $\bar{x}=\bar{x}_{k}=\frac{1}{P_{\mathrm{u}, k}^{(n)}(M-1) \beta_{k}}, \bar{y}=\bar{y}_{k}=$ $\sum_{i=1, i \neq k}^{K} P_{\mathrm{u}, i}^{(n)} \beta_{i}+1$, where $P_{\mathrm{u}, k}^{(n)}$ is the $n$-th feasible point of the constraints in (49). Clearly, the objective function (49) is non-concave function. To address the non-concave objective function (49), we follow an efficient relaxation technique by using the logarithm inequality [22], [23].

Note that, $f(x, y)=\ln \left(1+\frac{1}{x y}\right)$ is convex $\forall x, y \geqslant 0$ (the proof of this statement is followed by examining its Hessian), and hence, we have

$$
\begin{aligned}
& \ln \left(1+\frac{1}{x y}\right) \geq f(\bar{x}, \bar{y})+\langle\nabla f(\bar{x}, \bar{y}),(x, y)-(\bar{x}, \bar{y})\rangle \\
& =\ln \left(1+\frac{1}{\bar{x} \bar{y}}\right)+\frac{2}{\bar{x} \bar{y}+1}-\frac{1}{\bar{x}(\bar{x} \bar{y}+1)} x-\frac{1}{\bar{y}(\bar{x} \bar{y}+1)} y \\
& =a-b x+c y
\end{aligned}
$$

where $0<a=\ln \left(1+\frac{1}{\bar{x} \bar{y}}\right)+\frac{2}{\bar{x} \bar{y}+1}, 0<b=\frac{1}{\bar{x}(\bar{x} \bar{y}+1)}$, and $0<c=\frac{1}{\bar{y}(\bar{x} \bar{y}+1)}$.

From (49) and (50), we obtain

$$
F\left(P_{\mathrm{u}, k}\right) \geq F^{(n)}\left(P_{\mathrm{u}, k}\right)
$$


where

$$
\begin{aligned}
F^{(n)}\left(P_{\mathrm{u}, k}\right) & =\sum_{k=1}^{K}\left(a_{k}^{(n)}-b_{k}^{(n)} \frac{1}{P_{\mathrm{u}, k}(M-1) \beta_{k}}\right. \\
& \left.-c_{k}^{(n)} \sum_{\substack{i=1 \\
i \neq k}}^{K} P_{\mathrm{u}, i} \beta_{i}+1\right),
\end{aligned}
$$

and where $a_{k}^{(n)}=\ln \left(1+\frac{1}{\bar{x}_{k}^{(n)} \bar{y}_{k}^{(n)}}\right)+\frac{2}{\bar{x}_{k}^{(n)} \bar{y}_{k}^{(n)}+1}, b_{k}^{(n)}=$ $\frac{1}{\bar{x}_{k}^{(n)}\left(\bar{x}_{k}^{(n)} \bar{y}_{k}^{(n)}+1\right)}, c_{k}^{(n)}=\frac{1}{\bar{y}_{k}^{(n)}\left(\bar{x}_{k}^{(n)} \bar{y}_{k}^{(n)}+1\right)}$.

At the $n$-th iteration, the following convex program is solved to generate the next feasible point $P_{\mathrm{u}, k}^{(n+1)}$ of (49):

$$
\begin{aligned}
& \underset{P_{\mathrm{u}, k}}{\operatorname{maximize}} \frac{1}{\ln 2} F^{(n)}\left(P_{\mathrm{u}, k}\right), \\
& \text { s.t. }(48 b),(48 c)
\end{aligned}
$$

The initial point $P_{\mathrm{u}, k}^{(0)}$ can be easily determined by solving the convex problem as follows

$$
\underset{P_{\mathrm{u}, k}}{\operatorname{minimize}} \sum_{k=1}^{K} P_{\mathrm{u}, k}(M-1) \beta_{k} \text {, s.t. }(48 b),(48 c) .
$$

The algorithm to solve (48) is summarized in Algorithm 1.

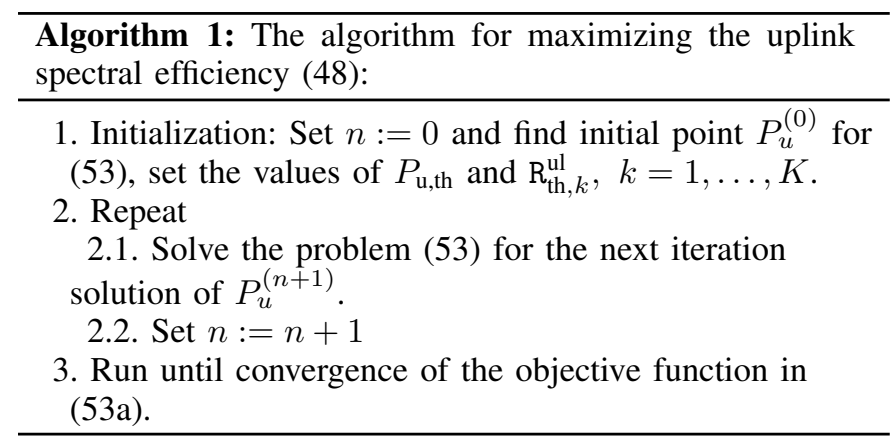

Complexity Analysis : The algorithm solving (48) involves $\varsigma=K$ real variables and $\varrho=3 K$ linear constraints. Thus, the per-iteration complexity of solving (48) is $\mathcal{C}=O\left(\varsigma^{2} \varrho^{2.5}+\right.$ $\left.\varrho^{3.5}\right)[22]$.

\section{B. Power Allocation Optimization at The Relay}

The power allocation optimization problem at the relay is formulated as

$$
\begin{aligned}
& \underset{\eta_{j(k, t)}^{(t)}}{\operatorname{maximize}} \sum_{k=1}^{K} \sum_{t=1}^{K-1} \tilde{\mathrm{R}}_{k}^{\mathrm{dl},(t)} \\
& \text { s.t. } \quad M \sum_{i=1}^{K} \eta_{j(i, t)}^{(t)} \beta_{i} \leqslant P_{\mathrm{r}, \mathrm{th}}, t=1, \ldots, t^{\prime} \\
& \tilde{\mathrm{R}}_{k}^{\mathrm{dl},(t)} \geq \mathrm{R}_{\mathrm{th}, k}^{\mathrm{dl},(t)}, k=1, \ldots, K, t=1, \ldots, K-1 .
\end{aligned}
$$

From (32) and (44), the power allocation problem is equivalent to

$$
\begin{aligned}
& \underset{\eta_{j(k, t)}^{(t)}}{\operatorname{maximize}} \sum_{k=1}^{K} \sum_{t=1}^{K-1} \log _{2} \\
& \times\left(\begin{array}{c}
o_{k} \eta_{j(k, t)}^{(t)}+v_{k} \sum_{i=1}^{t^{\prime}} \eta_{j(k, t)}^{\left(t^{\prime}-i+1\right)} \beta_{j\left(k, t-t^{\prime}+i-1\right)} \\
u_{k} \sum_{\substack{i=1 \\
j(i, t) \notin \mathcal{V}}, \eta_{k, t}}^{K} \eta_{j(i, t)}^{(t)} \beta_{i}+1
\end{array}\right)
\end{aligned}
$$

s.t. $\quad M \sum_{i=1}^{K} \eta_{j(i, t)}^{(t)} \beta_{i} \leqslant P_{\mathrm{r}, \mathrm{th}}, t=1, \ldots, t^{\prime}$

$$
\log _{2}\left(1+\frac{\eta_{j(k, t)}^{(t)}(M-1)(M-2) \beta_{k}^{2}}{(M-2) \beta_{k} \sum_{\substack{i=1 \\ j(i, t) \notin \mathcal{V}}}^{K} \eta_{j(i, t)}^{(t)} \beta_{i}+1}\right) \geq \mathrm{R}_{\mathrm{th}, k}^{\mathrm{dl},(t)},
$$

$\forall k, t=1, \ldots, t^{\prime}$

$$
\log _{2}\left(1+M \beta_{k} \sum_{i=1}^{t^{\prime}} \eta_{j(k, t)}^{\left(t^{\prime}-i+1\right)} \beta_{j\left(k, t-t^{\prime}+i-1\right)}\right) \geq \mathrm{R}_{\mathrm{th}, k}^{\mathrm{dl},(t)},
$$

$\forall k, t=t^{\prime}+1, \ldots, K-1$,

where

$$
\begin{aligned}
& o_{k}=\left\{\begin{array}{cc}
(M-1)(M-2) \beta_{k}^{2} & \text { if } t=1, \ldots, t^{\prime} \\
0 & \text { if } t>t^{\prime}
\end{array},\right. \\
& u_{k}=\left\{\begin{array}{ccc}
(M-2) \beta_{k} & \text { if } t=1, \ldots, t^{\prime}, \\
0 & \text { if } t>t^{\prime}
\end{array}\right. \\
& v_{k}=\left\{\begin{array}{ccc}
0 & \text { if } t=1, \ldots, t^{\prime} \\
M \beta_{k} & \text { if } & t>t^{\prime}
\end{array}\right.
\end{aligned}
$$

We define $x=x_{k, t}=\frac{1}{o_{k} \eta_{j(k, t)}^{(t)}+v_{k} \sum_{i=1}^{t^{\prime}} \eta_{j(k, t)}^{\left(t^{\prime}-i+1\right)} \beta_{j\left(k, t-t^{\prime}+i-1\right)}}$, $y=y_{k, t}=u_{k} \sum_{\substack{i=1 \\ j(i, t) \notin \mathcal{\nu}_{k, t}}}^{K} \eta_{j(i, t)}^{(t)} \beta_{i}+1$, and $\bar{x}=\bar{x}_{k, t}=$ $\frac{1}{o_{k}\left(\eta_{j(k, t)}^{(t)}\right)^{(n)}+v_{k} \sum_{i=1}^{t^{\prime}}\left(\eta_{j(k, t)}^{\left(t^{\prime}-i+1\right)}\right)^{(n)} \beta_{j\left(k, t-t^{\prime}+i-1\right)}}, \bar{y}=\bar{y}_{k, t}=$ $u_{k} \sum_{\substack{i=1 \\ j(i, t) \notin \mathcal{V}_{k, t}}}^{K}\left(\eta_{j(i, t)}^{(t)}\right)^{(n)} \beta_{i}+1$, where $\left(\eta_{j(k, t)}^{(t)}\right)^{(n)}$ is the $n$-th feasible point of the constraints in (56). We now apply a similar approach as in Section V-A to optimize the power allocation at the relay. At the $n$-th iteration, the following convex program is solved to determine the next feasible point 


$$
\begin{aligned}
& \left(\eta_{j(k, t)}^{(t)}\right)^{(n+1)} \text { of (56): } \\
& \underset{\eta_{j(k, t)}^{(t)}}{\operatorname{maximize}} \frac{1}{\ln 2} \bar{F}^{(n)}\left(\eta_{j(k, t)}^{(t)}\right), \\
& \text { s.t. } \quad M \sum_{i=1}^{K} \eta_{j(i, t)}^{(t)} \beta_{i} \leqslant P_{\mathrm{r}, \mathrm{th}}, t=1, \ldots, t^{\prime} \text {, } \\
& \beta_{k}^{2} \eta_{j(k, t)}^{(t)} \geq \frac{\left(2^{\mathrm{R}_{\mathrm{tl}, k}^{\mathrm{dl},(t)}}-1\right)}{(M-1)(M-2)} \\
& \times\left((M-2) \beta_{k} \sum_{\substack{i=1 \\
j(i, t) \notin \mathcal{V}_{k, t}}}^{K} \eta_{j(i, t)}^{(t)} \beta_{i}+1\right), \forall k, t=1, \ldots, t^{\prime}, \\
& M \beta_{k} \sum_{i=1}^{t^{\prime}} \eta_{j(k, t)}^{\left(t^{\prime}-i+1\right)} \beta_{j\left(k, t-t^{\prime}+i-1\right)} \geq 2^{\mathrm{R}_{\mathrm{th}, k}^{\mathrm{dl},(t)}}-1, \\
& \forall k, t=t^{\prime}+1 \ldots, K-1 \text {, }
\end{aligned}
$$

where $\bar{F}^{(n)}\left(\eta_{j(k, t)}^{(t)}\right)$ is given (59) at the top of the next page, and where $\bar{a}_{k}^{(n)}=\ln \left(1+\frac{1}{\bar{x}_{k}^{(n)} \bar{y}_{k}^{(n)}}\right)+\frac{2}{\bar{x}_{k}^{(n)} \bar{y}_{k}^{(n)}+1}$, $\bar{b}_{k}^{(n)}=\frac{1}{\bar{x}_{k}^{(n)}\left(\bar{x}_{k}^{(n)} \bar{y}_{k}^{(n)}+1\right)}, \bar{c}_{k}^{(n)}=\frac{1}{\bar{y}_{k}^{(n)}\left(\bar{x}_{k}^{(n)} \bar{y}_{k}^{(n)}+1\right)}$.

Note that, the initial point $\left(\eta_{j(k, t)}^{(t)}\right)^{(0)}$ can be easily determined by solving the following convex problem:

$$
\begin{aligned}
& \underset{\substack{\eta_{j(k, t)}^{(t)} \\
\operatorname{minimize}}}{K} \sum_{k=1}^{K} \sum_{t=1}^{K-1} \\
& \times\left(o_{k} \eta_{j(k, t)}^{(t)}+v_{k} \sum_{i=1}^{t^{\prime}} \eta_{j(k, t)}^{\left(t^{\prime}-i+1\right)} \beta_{j\left(k, t-t^{\prime}+i-1\right)}\right), \\
& \text { s.t. } \quad M \sum_{i=1}^{K} \eta_{j(i, t)}^{(t)} \beta_{i} \leqslant P_{\mathrm{r}, \mathrm{th}}, t=1, \ldots, t^{\prime}, \\
& \beta_{k}^{2} \eta_{j(k, t)}^{(t)} \geq \frac{\left(2^{\mathrm{R}_{\mathrm{th}, k}^{\mathrm{dl},(t)}}-1\right)}{(M-1)(M-2)} \\
& \times\left((M-2) \beta_{k} \sum_{\substack{i=1 \\
j(i, t) \notin \mathcal{V}_{k, t}}}^{K} \eta_{j(i, t)}^{(t)} \beta_{i}+1\right), \forall k, t=1, \ldots, t^{\prime}, \\
& M \beta_{k} \sum_{i=1}^{t^{\prime}} \eta_{j(k, t)}^{\left(t^{\prime}-i+1\right)} \beta_{j\left(k, t-t^{\prime}+i-1\right)} \geq 2^{\mathrm{R}_{\mathrm{tl}, k}^{\mathrm{d},(t)}}-1, \\
& \forall k, t=t^{\prime}+1, \ldots, K-1 \text {. }
\end{aligned}
$$

The steps to solve (55) are summarized in Algorithm 2.

Complexity Analysis: The algorithm for maximizing problem (56) involves $\varsigma=K\left\lceil\frac{K-1}{2}\right\rceil$ real variables and $\varrho=$ $\left\lceil\frac{K-1}{2}\right\rceil+K^{2}$ linear constraints. Thus, the per-iteration complexity of solving (56) is $\mathcal{C}=O\left(\varsigma^{2} \varrho^{2.5}+\varrho^{3.5}\right)$.

\section{ENERGY EFFICIENCY MAXIMIZATION}

In this section, we elaborate on the energy efficiency of our proposed system model taking into account the hardware

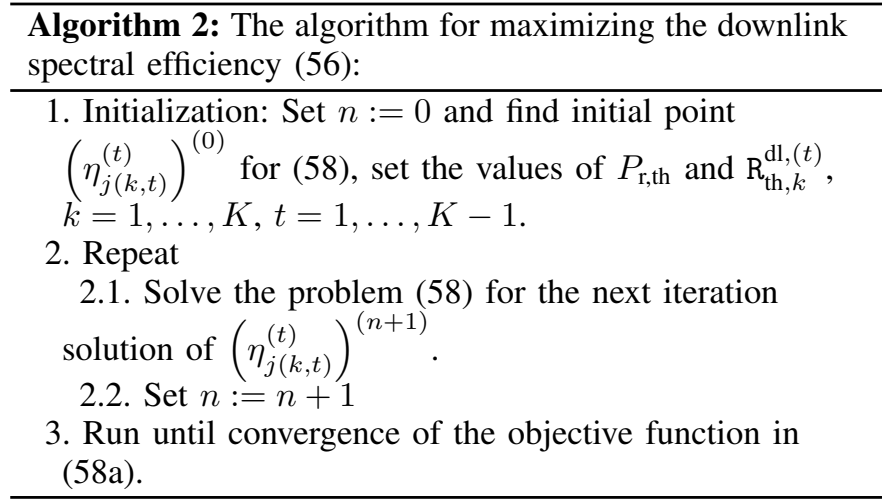

power consumption. We propose a novel power control algorithm which maximizes the energy efficiency, subject to a given per-user spectral efficiency and the power constraints of each user/relay.

To begin with, the energy efficiency (bit/Joule) is determined as the ratio between the sum throughput (in bit), given by $B \tilde{\mathrm{SE}}_{\text {sum }}$, to the total power consumption (Watt). More precisely, the energy efficiency is given by [24]

$$
\begin{aligned}
& \text { EE } \\
& =\frac{B \tilde{\mathrm{SE}}_{\mathrm{sum}}}{\sum_{k=1}^{K} \frac{P_{\mathrm{u}, k}}{\zeta_{\mathrm{u}, k}}+\frac{M}{\zeta_{\mathrm{r}}} \sum_{t=1}^{t^{\prime}} \sum_{k=1}^{K} \beta_{k} \eta_{j(k, t)}^{(t)}+B P_{\mathrm{LD}} \tilde{\mathrm{SE}}_{\mathrm{sum}}+P_{\mathrm{LID}}},
\end{aligned}
$$

where $B$ is the transmission bandwidth, $0<\zeta_{\mathrm{u}, j(k, t)} \leq 1$ and $0<\zeta_{\mathrm{r}} \leq 1$ are the power amplifier efficiencies of each user and the relay, respectively. Furthermore, $P_{\mathrm{LD}}$ (in Watt/(bit/s)) represents the load dependent power including power for signal processing (coding and decoding) and the power consumption of the backhaul traffic, and $P_{\text {LID }}$ (in Watt) accounts for the load independent power including the power required to run the circuit components at the relay and each user, and the fixed power consumption for the backhaul link between the relay and the core network. As a consequence, the power allocation problem for the energy efficiency is now formulated as

$$
\begin{aligned}
& \underset{P_{\mathrm{u}, k}, \eta_{j(k, t)}^{(t)}}{\operatorname{maximize}} \mathrm{EE} \\
& \text { s.t. } \quad \tilde{\mathrm{SE}}_{\mathrm{sum}}=\tilde{\mathrm{SE}}_{\mathrm{th}}, \\
& 0 \leq P_{\mathrm{u}, k} \leq P_{\mathrm{u}, \mathrm{th}}, k=1, \ldots, K, \\
& 0 \leq \sum_{k=1}^{K} M \eta_{j(k, t)}^{(t)} \beta_{k} \leq P_{\mathrm{r}, \mathrm{th}}, t=1, \ldots, t^{\prime},
\end{aligned}
$$

where $\tilde{\mathrm{SE}}_{\mathrm{th}}$ is the required spectral efficiency of the system. In our optimization problem, we want to allocate the powers to the users and the relay to maximize the energy efficiency for a given quality-of-service, i.e. sum spectral efficiency. This will provide a fair comparison among different schemes since they will have the same sum spectral efficiency. This is why the constraint in $(62 b)$ is satisfied with equality. 


$$
\begin{aligned}
& \bar{F}^{(n)}\left(\eta_{j(k, t)}^{(t)}\right)=\sum_{k=1}^{K} \sum_{t=1}^{K-1}\left(\bar{a}_{k}^{(n)}-\bar{b}_{k}^{(n)} \bar{x}-\bar{c}_{k}^{(n)} \bar{y}\right) \\
& =\sum_{k=1}^{K} \sum_{t=1}^{K-1}\left(\bar{a}_{k}^{(n)}-\bar{b}_{k}^{(n)} \frac{1}{o_{k} \eta_{j(k, t)}^{(t)}+v_{k} \sum_{i=1}^{t^{\prime}} \eta_{j(k, t)}^{\left(t^{\prime}-i+1\right)} \beta_{j\left(k, t-t^{\prime}+i-1\right)}}-\bar{c}_{k}^{(n)}\left(u_{k} \sum_{\substack{i=1 \\
j(i, t) \notin \mathcal{V} \\
k, t}}^{K} \eta_{j(i, t)}^{(t)} \beta_{i}+1\right)\right),
\end{aligned}
$$

By dividing the numerator and denominator of $\mathrm{EE}$ by $B \widetilde{S E}_{\text {sum }}$, the objective function (62a) can be rewritten as

$$
\begin{aligned}
& \mathrm{EE} \\
& =\frac{1}{\frac{\sum_{k=1}^{K} \frac{1}{\zeta_{\mathrm{u}, k}} P_{\mathrm{u}, k}+\frac{1}{\zeta_{\mathrm{r}}} \sum_{t=1}^{t^{\prime}} \sum_{k=1}^{K} M \beta_{k} \eta_{j(k, t)}^{(t)}+P_{\mathrm{LID}}}{B \widetilde{\mathrm{SE}}_{\mathrm{sum}}}+P_{\mathrm{LD}}} .
\end{aligned}
$$

It can be clearly seen from (63) that in order to maximize EE, we can minimize the first term of the denominator (63). As a consequence, the problem (62) is equivalent to

$$
\begin{aligned}
& \underset{P_{\mathrm{u}, k}, \eta_{j(k, t)}^{(t)}}{\operatorname{minimize}} \sum_{k=1}^{K} \frac{1}{\zeta_{\mathrm{u}, k}} P_{\mathrm{u}, k}+\frac{M}{\zeta_{\mathrm{r}}} \sum_{t=1}^{t^{\prime}} \sum_{k=1}^{K} \beta_{k} \eta_{j(k, t)}^{(t)} \\
& \text { s.t. } \quad(62 c),(62 d) \\
& \frac{1}{t^{\prime}+1} \sum_{k=1}^{K} \sum_{t=1}^{K-1} \log _{2}\left(1+\min \left(\gamma_{j(k, t)}^{\mathrm{ul}}, \gamma_{k}^{\mathrm{dl},(t)}\right)\right)=\tilde{\mathrm{SE}}_{\mathrm{th}}
\end{aligned}
$$

where

$$
\begin{aligned}
& \gamma_{j(k, t)}^{\mathrm{ul}}= \frac{e_{k, t} P_{\mathrm{u}, j(k, t)}}{\sum_{i=1, i \neq k}^{K} \beta_{i} P_{\mathrm{u}, j(i, t)}+1}, \\
& \gamma_{k}^{\mathrm{dl},(t)}=\left\{\begin{array}{c}
\frac{f_{k, t} \eta_{j(k, t)}^{(t)}}{q_{k, t} \sum_{i=1}^{K} \beta_{i} \eta_{j(i, t)}^{(t)}+1} \text { if } t=1, \ldots, t^{\prime} \\
j(i, t) \notin \mathcal{V}_{k, t} \\
M \beta_{k} \sum_{i=1}^{t^{\prime}} \eta_{j(k, t)}^{\left(t^{\prime}-i+1\right)} \beta_{j\left(k, t-t^{\prime}+i-1\right)} \\
\text { if } t=t^{\prime}+1, \ldots, K-1
\end{array}\right.
\end{aligned}
$$

and where $e_{k, t}=(M-1) \beta_{j(k, t)}, f_{k, t}=(M-1)(M-$ 2) $\beta_{k}^{2}, q_{k, t}=(M-2) \beta_{k}$.

The problem (64) is further equivalent to

$$
\begin{aligned}
& \underset{P_{\mathrm{u}, k}, \eta_{j(k, t)}^{(t)}, \gamma_{k, t}}{\operatorname{minimize}}(64 a) \\
& \text { s.t. } \quad \frac{1}{t^{\prime}+1} \sum_{k=1}^{K} \sum_{t=1}^{K-1} \log _{2}\left(1+\gamma_{k, t}\right)=\tilde{\mathrm{SE}}_{\mathrm{th}}, \\
& \gamma_{k, t} \leq \gamma_{j(k, t)}^{\mathrm{ul}}, k=1, \ldots, K, t=1, \ldots, K-1, \\
& \gamma_{k, t} \leq \gamma_{k}^{\mathrm{dl},(t)}, k=1, \ldots, K, t=1, \ldots, K-1, \\
& (62 c),(62 d) .
\end{aligned}
$$

Since $e_{k, t}, f_{k, t}$, and $q_{k, t}$ are positive numbers, (67) can be equivalently written as

$$
\begin{aligned}
& \underset{P_{\mathrm{u}, j(k, t)}, \eta_{j(k, t)}^{(t)}, \gamma_{k, t}}{\operatorname{minimize}}(64 a) \\
& \text { s.t. } \prod_{k=1}^{K} \prod_{t=1}^{K-1}\left(1+\gamma_{k, t}\right)=2^{\left(t^{\prime}+1\right) \tilde{\mathrm{SE}}_{\mathrm{th}}}, \\
& \frac{\gamma_{k, t}}{e_{k, t}} \sum_{i=1, i \neq k}^{K} \beta_{i} P_{\mathrm{u}, j(i, t)}\left(P_{\mathrm{u}, j(k, t)}\right)^{-1}+\frac{\gamma_{k, t}}{e_{k, t}}\left(P_{\mathrm{u}, j(k, t)}\right)^{-1} \leq 1,
\end{aligned}
$$

$k=1, \ldots, K, t=1, \ldots, K-1$,

$\frac{\gamma_{k, t} q_{k, t}}{f_{k, t}}\left(\eta_{j(k, t)}^{(t)}\right)^{-1} \sum_{\substack{i=1 \\ j(i, t) \notin \mathcal{V}_{k, t}}}^{K} \beta_{i} \eta_{j(i, t)}^{(t)}+\frac{\gamma_{k, t}}{f_{k, t}}\left(\eta_{j(k, t)}^{(t)}\right)^{-1} \leq 1$,

$k=1, \ldots, K, t=1, \ldots, t^{\prime}$,

$\frac{\gamma_{k, t}}{t^{\prime} M \beta_{k}} \prod_{i=1}^{t^{\prime}}\left(\eta_{j(k, t)}^{\left(t^{\prime}-i+1\right)} \beta_{j\left(k, t-t^{\prime}+i-1\right)}\right)^{-1 / t^{\prime}} \leq 1$,

$k=1, \ldots, K, t=t^{\prime}+1, \ldots, K-1$,

$(62 c),(62 d)$.

We can see that the most challenging constraint in (68) is (68b). If the equality function (68b) is a posynomial function, then problem (68) will be a geometric program (GP) which can be solved effectively by using standard convex optimization tools such as CVX [25] or GGPLAB [26]. However, (68b) is not a posynomial function, and hence, the problem (68) cannot be solved directly via the above tools. To deal with this, we use techniques in [27], [28] by finding an approximate solution of (68) through solving a sequence of GPs. With this technique, we approximate $\left(1+\gamma_{k, t}\right)$ to $\kappa_{k, t} \gamma_{k, t}^{\xi_{k, t}}$ near a point $\hat{\gamma}_{k, t}>0$, where $\xi_{k, t} \triangleq \frac{\hat{\gamma}_{k, t}}{1+\hat{\gamma}_{k, t}}$ and $\kappa_{k, t} \triangleq \hat{\gamma}_{k, t}^{-\xi_{k, t}}\left(1+\hat{\gamma}_{k, t}\right)$. Consequently, near the point $\hat{\gamma}_{k, t}>0$, we obtain the following approximation:

$$
\prod_{k=1}^{K} \prod_{t=1}^{K-1}\left(1+\gamma_{k, t}\right) \approx \prod_{k=1}^{K} \prod_{t=1}^{K-1} \kappa_{k, t} \gamma_{k, t}^{\xi_{k, t}}
$$

which is a monomial function. Therefore, by using the above approximation, the constraint (68b) is a posynomial function, and hence, the optimization problem (68) becomes a GP. As a result, problem (68) can be solved iteratively via solving a sequences of GPs. The successive approximation algorithm to solve (68) is summarized in Algorithm 3.

Complexity Analysis: The successive approximation algorithm for solving problem (68) involves $\varsigma=2 K\left\lceil\frac{K-1}{2}\right\rceil+$ $K(K+1)$ real variables, $\varrho=\left\lceil\frac{K-1}{2}\right\rceil+2 K$ linear constraints, and $\rho=2 K(K-1)+1$ geometric constraints. 


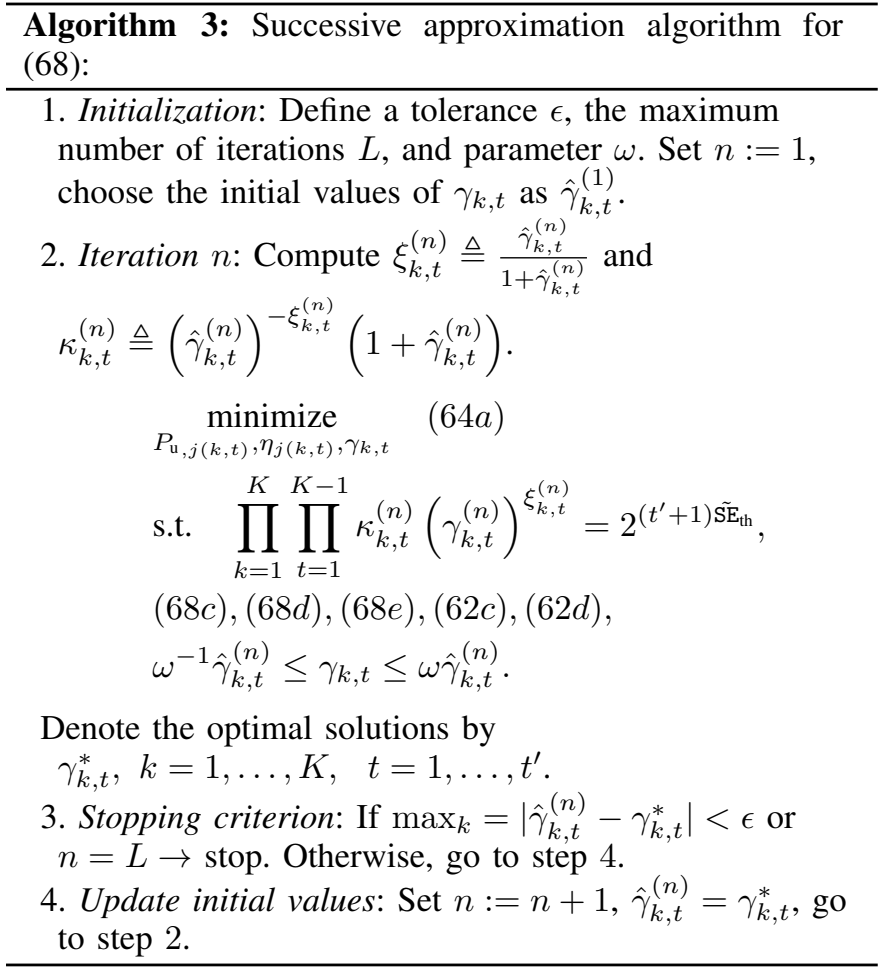

Therefore, the per-iteration complexity of solving (68) is $\mathcal{C}=O\left(\varsigma^{2}(\varrho+\rho)^{2.5}+(\varrho+\rho)^{3.5}\right)$.

\section{IMPERFECT CSI ANALYSIS}

We will now delineate the mathematical analysis for the more practical case of imperfect CSI. Due to space constraints, most results are provided without proof. For simplicity, with imperfect CSI, we neglect the power control. More precisely, we assume $P_{\mathrm{u}, k}=P_{\mathrm{u}}$ for all $k$, and that $\eta_{j(k, t)}^{(t)}$ are the same for all $t$ and $k$.

\section{A. Channel Estimation}

The channels and the effective channel gains need to be estimated at the relay and the users. This can be done via the training phase.

1) Channel Estimation at the Relay: All $K$ users simultaneously transmit their pilot sequences of $\tau$ symbols to the relay. We assume that the pilots sequences of $K$ users are pairwisely orthogonal. This requires that $\tau \geq K$. Furthermore, we denote $P_{\mathrm{u}, \mathrm{p}}$ as the transmit power of each pilot symbol. Then, the MMSE channel estimate of $\mathbf{G}$ is [29]

$$
\hat{\mathbf{G}}=\mathbf{G}-\mathbf{E},
$$

where $\mathbf{E}$ is the channel estimation error matrix which is independent of $\hat{\mathbf{G}}$. In addition, we have $\hat{\mathbf{G}} \sim \mathcal{C N}(0, \hat{\mathbf{D}})$ and $\mathbf{E} \sim \mathcal{C N}\left(0, \mathbf{D}_{E}\right)$, where $\hat{\mathbf{D}}$ and $\mathbf{D}_{E}$ are diagonal matrices whose $(k, k)$-th components are $\hat{\sigma}_{k}^{2}=\frac{\tau P_{\mathrm{u}, \mathrm{p}} \beta_{k}^{2}}{\tau P_{\mathrm{u}, \mathrm{p}} \beta_{k}+1}$ and $\hat{\sigma}_{e, k}^{2}=\beta_{k}-\hat{\sigma}_{k}^{2}$, respectively.

2) Channel Estimation at each User: The $k$-th user needs to know the effective channel gains $\theta_{k i}=\mathbf{g}_{k}^{H} \hat{\mathbf{g}}_{i}, i=1, \ldots, K$, to implement our proposed scheme. These effective channel gains can be estimated through downlink training. Since the users need to estimate only the effective channel gains, the downlink channel overhead is independent of the number of relay antennas.

In the downlink training phase, the relay beamforms $K$ orthogonal pilot sequences to all users using the channel estimates acquired in the uplink training phase. Following the same technique used in [17], the estimates of $\theta_{k k}$ and $\theta_{k i}$ are, respectively, given by

$$
\begin{aligned}
\hat{\theta}_{k k} & =\left(\theta_{k k}+\frac{n_{p, k}}{\sqrt{\alpha}}-M \hat{\sigma}_{k}^{2}\right) \\
& \times\left(\frac{1}{\alpha M \hat{\sigma}_{k}^{2} \beta_{k}}+1\right)^{-1}+M \hat{\sigma}_{k}^{2},
\end{aligned}
$$

and

$$
\hat{\theta}_{k i}=\left(\theta_{k i}+\frac{n_{p, i}}{\sqrt{\alpha}}\right)\left(\frac{1}{\alpha M \hat{\sigma}_{i}^{2} \beta_{k}}+1\right)^{-1},
$$

where $n_{p, i}, i=1, \ldots, K$ are Gaussian noises with zero mean and unit variance, and $\alpha$ is a normalization constant chosen so that the power of each pilot symbol is $P_{\mathrm{r}, \mathrm{p}}$. Hence,

$$
\alpha=\frac{\tau P_{\mathrm{r}, \mathrm{p}}}{\mathbb{E}\left\{\operatorname{Tr}\left(\hat{\mathbf{G}} \hat{\mathbf{G}}^{H}\right)\right\}} .
$$

\section{B. Data Transmission}

Similarly to the case of perfect CSI, the data transmission includes multiple-access phase and broadcast phase. The main difference is that the true channels are replaced by their channel estimates.

1) Multiple-Access Phase:

After receiving data from the $K$ users, the relay treats the channel estimate as the true channel and uses MRC to decode the signal transmitted from the $K$ users as $\mathbf{r}^{\mathrm{im}}=\hat{\mathbf{G}}^{H} \mathbf{y}_{\mathrm{R}}$. Thus, the uplink spectral efficiency $\mathrm{R}_{k}^{\mathrm{ul}, i m}$ of the $k$-th user is given (75) at the top of the next page.

2) Broadcast Phase:

\section{- Conventional Transmission Protocol:}

The transmitted signal vector at the relay in the $t$-th timeslot is given by

$$
\mathbf{x}_{r}^{(t)}=\sqrt{\alpha_{1}} \sum_{i=1}^{K} \hat{\mathbf{g}}_{i} x_{j(i, t)},
$$

where $\alpha_{1}$ is the normalization factor power which is chosen to meet a long-term power constraint at the relay: $\mathbb{E}\left\{\left\|\mathbf{x}_{r}^{(t)}\right\|^{2}\right\}=P_{\mathrm{r}}$. Thus, we obtain

$$
\alpha_{1}=\frac{P_{\mathrm{r}}}{\sum_{i=1}^{K} \mathbb{E}\left\{\operatorname{Tr}\left(\hat{\mathbf{g}}_{i} \hat{\mathbf{g}}_{i}^{H}\right)\right\}},
$$

The downlink spectral efficiency of the $k$-th user in the $t$-th time-slot is

$$
=\log _{2}\left(1+\frac{\alpha_{1}^{\mathrm{dl}, \mathrm{im},(t)}\left|\mathbb{E}\left\{\mathbf{g}_{k}^{H} \hat{\mathbf{g}}_{k}\right\}\right|^{2}}{\alpha_{1} \operatorname{Var}\left(\mathbf{g}_{k}^{H} \hat{\mathbf{g}}_{k}\right)+\alpha_{1} \sum_{\substack{i=1 \\ j(i, t) \neq j(k, t)}}^{K} \mathbb{E}\left\{\left|\mathbf{g}_{k}^{H} \hat{\mathbf{g}}_{i}\right|^{2}\right\}+1}\right) .
$$




$$
\mathrm{R}_{k}^{\mathrm{ul}, \mathrm{im}}=\log _{2}\left(1+\frac{P_{\mathrm{u}}\left|\mathbb{E}\left\{\hat{\mathbf{g}}_{k}^{H} \mathbf{g}_{k}\right\}\right|^{2}}{P_{\mathrm{u}} \mathbb{V} a r\left(\hat{\mathbf{g}}_{k}^{H} \mathbf{g}_{k}\right)+P_{\mathrm{u}} \sum_{\substack{i=1 \\ i \neq k}}^{K} \mathbb{E}\left\{\left|\hat{\mathbf{g}}_{k}^{H} \mathbf{g}_{i}\right|^{2}\right\}+\mathbb{E}\left\{\left|\hat{\mathbf{g}}_{k}^{H} \mathbf{n}_{\mathrm{R}}\right|^{2}\right\}}\right) .
$$

From (75) and (78) the sum spectral efficiency of the conventional scheme is

$$
\mathrm{SE}_{\text {sum }}^{\mathrm{im}}=\frac{1}{K}\left(\frac{T-\tau}{T}\right) \sum_{k=1}^{K} \sum_{t=1}^{K-1} \min \left(\mathrm{R}_{j(k, t)}^{\mathrm{ul}, \mathrm{im}}, \mathrm{R}_{k}^{\mathrm{dl}, \mathrm{im},(t)}\right) \text {. }
$$

\section{- Proposed Transmission Protocol:}

The signal vector transmitted from the relay at the $t$-th timeslot is

$$
\mathbf{s}^{(t)}=\sqrt{\alpha_{2}} \sum_{i=1}^{K} \hat{\mathbf{g}}_{i} x_{j(i, t)},
$$

where $\alpha_{2}$ is chosen to satisfy $\mathbb{E}\left\{\left\|\mathbf{s}^{(t)}\right\|^{2}\right\}=P_{\mathrm{r}}$ which is equivalent to $\alpha_{2}=\frac{P_{\mathrm{r}}}{\sum_{i=1}^{K} \mathbb{E}\left\{\operatorname{Tr}\left(\hat{\mathbf{g}}_{i} \hat{\mathbf{g}}_{i}^{H}\right)\right\}}$.

Then, the signal received at the $k$-th user is

$$
\begin{aligned}
y_{u, k}^{(t)} & =\mathbf{g}_{k}^{H} \mathbf{s}^{(t)}+n_{u, k}^{(t)}=\sqrt{\alpha_{2}} \sum_{i=1}^{K} \mathbf{g}_{k}^{H} \hat{\mathbf{g}}_{i} x_{j(i, t)}+n_{u, k}^{(t)} \\
& =\sqrt{\alpha_{2}} \sum_{i=1}^{K} \theta_{k i} x_{j(i, t)}+n_{u, k}^{(t)} .
\end{aligned}
$$

At the $t$-th time-slot, the $k$-th user detects $x_{j(k, t)}$ using the channel estimate $\hat{\theta}_{k k}$ in (72) and the knowledge of $\left\{x_{j(k-1,1)}, x_{j(k, 1)}, x_{j(k, 2)}, \ldots, x_{j(k, t-1)}\right\}$. The received signal at the $k$-th user after interference cancellation is

$$
\tilde{y}_{u, k}^{(t)}=y_{u, k}^{(t)}-\sqrt{\alpha_{2}} \sum_{\substack{i=1 \\ j(i, t) \in \mathcal{V}_{k, t} \backslash j(k, t)}}^{K} \hat{\theta}_{k i} x_{j(i, t)},
$$

and hence, the spectral efficiency $\mathrm{R}_{k}^{\mathrm{d} l \mathrm{im},(t)}$ at user $k$ in the $t$-th time-slot can be expressed as in (83) at the top of the next page.

After $t^{\prime}=\left\lceil\frac{K-1}{2}\right\rceil$ time-slots, the same technique as in the perfect CSI case is used. Denote by

$$
\overline{\mathbf{n}}_{k}^{\left(t^{\prime}\right)} \triangleq\left[\begin{array}{c}
\sqrt{\alpha_{2}} \sum_{i=1}^{K} \epsilon_{k j(k, K-k+i-1)} x_{j(k, i-1)}+n_{k, 1}^{\left(t^{\prime}\right)} \\
\sqrt{\alpha_{2}} \sum_{i=1}^{K} \epsilon_{k j(k, K-k+i-2)} x_{j(k, i-1)}+n_{k, 2}^{\left(t^{\prime}\right)} \\
\vdots \\
\sqrt{\alpha_{2}} \sum_{i=1}^{K} \epsilon_{k j\left(k, K-k+i-t^{\prime}\right)} x_{j(k, i-1)}+n_{k, t^{\prime}}^{\left(t^{\prime}\right)}
\end{array}\right],
$$

and

$$
\mathbf{A}_{k}^{\left(t^{\prime}\right)} \triangleq \sqrt{\alpha_{2}}\left[\begin{array}{cccc}
\hat{\theta}_{k j(k, 1)} & \hat{\theta}_{k j(k, 2)} & \ldots & \hat{\theta}_{k j\left(k, K-t^{\prime}-1\right)} \\
\hat{\theta}_{k j(k, 2)} & \hat{\theta}_{k j(k, 3)} & \ldots & \hat{\theta}_{k j\left(k, K-t^{\prime}\right)} \\
\vdots & \vdots & & \vdots \\
\hat{\theta}_{k j\left(k, t^{\prime}\right)} & \hat{\theta}_{k j\left(k, t^{\prime}+1\right)} & \ldots & \hat{\theta}_{k j(k, K-2)}
\end{array}\right] .
$$

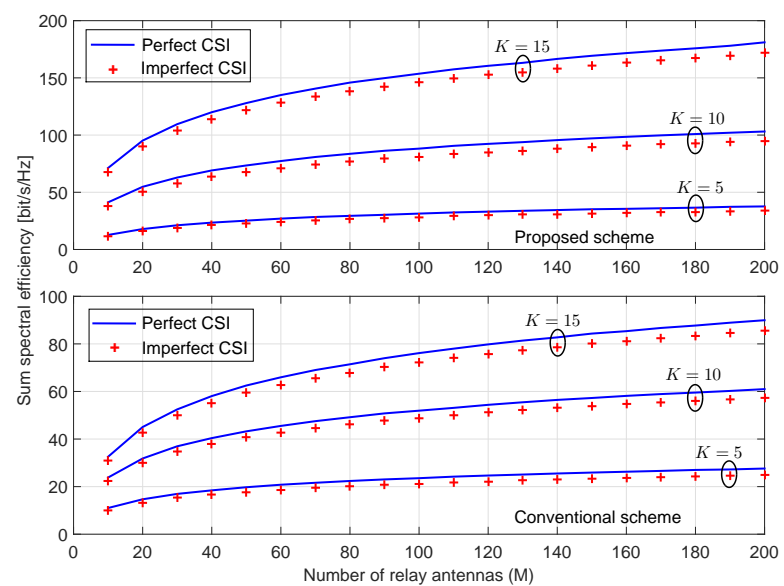

Fig. 1: The comparison between the perfect and imperfect CSI of the sum spectral efficiency versus the number of relay antennas for the conventional and proposed schemes with different $K$. We choose $P_{\mathrm{r}}=P_{\mathrm{r}, \mathrm{p}}=10 \mathrm{~dB}, P_{\mathrm{u}}=P_{\mathrm{p}}=0$ $\mathrm{dB}, T=200, \tau=K$ and $\beta_{k}=1, \forall k=1, \ldots, K$.

Then, we can obtain the spectral efficiency of the $k$-th user as

$$
\mathrm{R}_{k}^{\mathrm{dl}, \text {,im },\left(t^{\prime}+n\right)}=\log _{2}\left(1+\frac{\alpha_{2}}{\mathbb{E}\left\{\left|\mathbf{z}_{n}^{T} \overline{\mathbf{n}}_{k}^{\left(t^{\prime}\right)}\right|^{2}\right\}}\right),
$$

where

$$
\mathbf{Z}^{T} \triangleq\left(\left(\mathbf{A}_{k}^{\left(t^{\prime}\right)}\right)^{H} \mathbf{A}_{k}^{\left(t^{\prime}\right)}\right)^{-1}\left(\mathbf{A}_{k}^{\left(t^{\prime}\right)}\right)^{H} .
$$

From (75), (83) and (86), the sum spectral efficiency of the proposed scheme is

$$
\begin{aligned}
\mathrm{SE}_{\text {sum }}^{\mathrm{im}} & =\left(\frac{1}{t^{\prime}+1}\right)\left(\frac{T-2 \tau}{T}\right) \\
& \times \sum_{k=1}^{K} \sum_{t=1}^{K-1} \min \left(\mathrm{R}_{j(k, t)}^{\mathrm{ul}, \mathrm{im}}, \mathrm{R}_{k}^{\mathrm{dl}, \mathrm{im},(t)}\right) .
\end{aligned}
$$

Unfortunately, the derivation of closed-form expressions for the SE is very challenging, if not impossible, once we move away from the perfect CSI assumption.

Figure 1 shows the comparison of the sum spectral efficiencies for perfect and imperfect CSI. As expected, due to the the imperfect channel estimation and the channel estimation overhead, the spectral efficiency of imperfect CSI is lower than the one of perfect CSI. More importantly, even the value of powers is moderate and the length of training duration equals the number of users, the performance gap between imperfect and perfect CSI cases is small. 


$$
\mathrm{R}_{k}^{\mathrm{dl}, \text { im },(t)}=\log _{2}\left(1+\frac{\alpha_{2}\left|\mathbb{E}\left(\hat{\theta}_{k k}\right)\right|^{2}}{\alpha_{2} \mathbb{V} a r\left(\hat{\theta}_{k k}\right)+\alpha_{2} \sum_{\substack{i=1 \\ j(i, t) \notin \mathcal{V}}}^{K} \mathbb{E}\left\{\left|\hat{\theta}_{k i}\right|^{2}\right\}+\alpha_{2} \sum_{i=1}^{K} \mathbb{E}\left\{\left|\epsilon_{k i}\right|^{2}\right\}+1}\right) .
$$

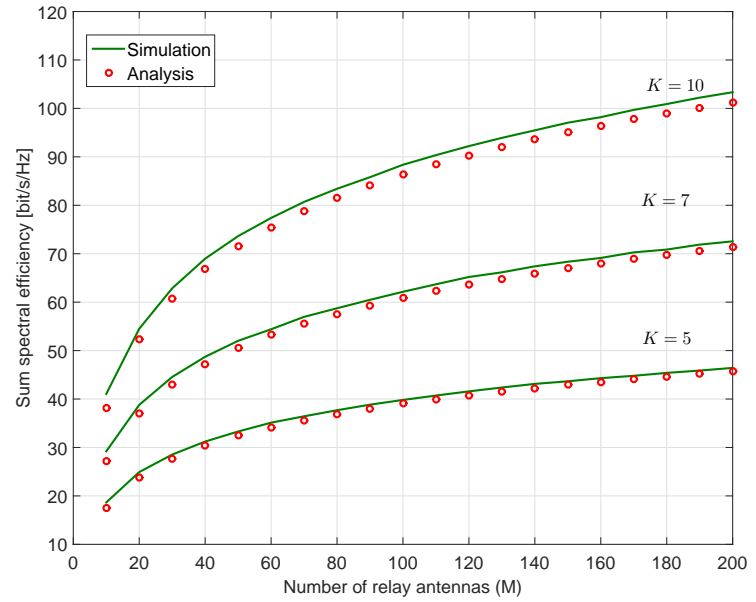

Fig. 2: The sum spectral efficiency versus the number of relay antennas with different $K$. We choose $P_{\mathrm{r}, \mathrm{th}}=10 \mathrm{~dB}, P_{\mathrm{u}, k}=$ $P_{\mathrm{u}, \mathrm{th}}=0 \mathrm{~dB}$, and $\beta_{k}=1, \forall k=1, \ldots, K$.

\section{NUMERICAL RESULTS}

In this section, a set of results are provided to evaluate the performance of the system designs (transmission protocol, as well as, power allocation schemes) proposed in the previous sections. Our performance measures are the sum spectral efficiency given by (45) and the energy efficiency given by (61).

\section{A. Scenario I}

To get initial insights on the tightness of our closedform expressions, the benefits of our proposed transmission protocols, as well as, the power allocation algorithms, we consider a simple scenario where $\beta_{k}=1$, for $k=1, \ldots, K$, and $\eta_{j(i, t)}^{(t)}=P_{\mathrm{r}, \mathrm{th}} /\left(M \sum_{i=1}^{K} \beta_{i}\right)$ for all $i$ and $t$.

Our first objective is to validate the sum spectral efficiency given by (45). Figure 2 shows the sum spectral efficiency of our proposed scheme versus the number of relay antennas with different numbers of users $K$. The "simulation" curves are generated from the outputs of a Monte-Carlo simulator using (6), (31), and (43), while the "analysis" curves represent our analytical results obtained by using the lower bounds (7), (32), and the asymptotic result (44). In this example, we choose $P_{\mathrm{u}, k}=P_{\mathrm{u}, \mathrm{th}}=0 \mathrm{~dB}$, and $P_{\mathrm{r}, \mathrm{th}}=10 \mathrm{~dB}$. We can see that the simulated sum spectral efficiency and our analytical results match perfectly, even at small number of users $K$. This verifies the tightness of our closed-form expressions. Moreover, when the number of antennas $M$ increases, the sum spectral efficiency grows noticeably.

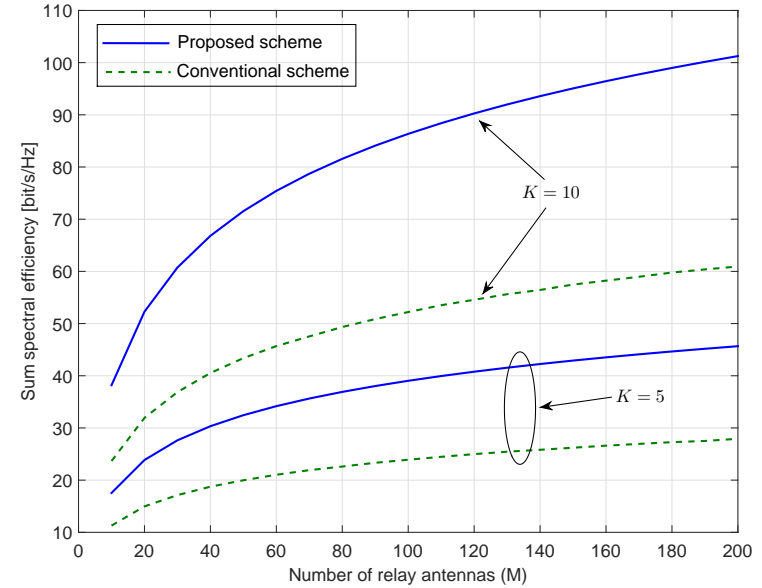

Fig. 3: The sum spectral efficiency of the proposed and the conventional scheme versus the number of relay antennas with $K=5$, and $K=10$. We choose $P_{\mathrm{u}, k}=P_{\mathrm{u}, \mathrm{th}}=0 \mathrm{~dB}$, $P_{\mathrm{r}, \mathrm{th}}=10 \mathrm{~dB}$, and $\beta_{k}=1, \forall k=1, \ldots, K$.

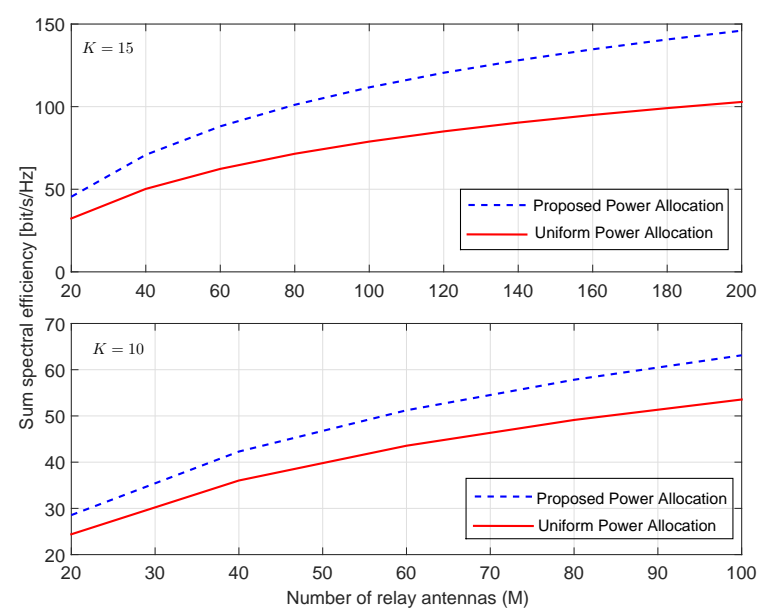

Fig. 4: The comparison of sum spectral efficiency between the proposed and uniform power allocation schemes for $K=10$ and 15 . We choose $\beta_{k}=1, \forall k=1, \ldots, K$.

Next, we compare the sum spectral efficiency of the proposed transmission protocol discussed in Section IV with that of the conventional scheme discussed in Section III. Figure 3 shows the sum spectral efficiency versus the number of relay antennas for $K=5$ and 10 . The sum spectral efficiency of our proposed scheme is given by (45) and the 
TABLE I:

SYSTEM PARAMETERS FOR THE SIMULATION FOR FIG. 5

\begin{tabular}{|c|c|}
\hline Parameters & Values \\
\hline Transmission bandwidth $B$ & $20 \mathrm{MHz}$ \\
The load dependent power $P_{\mathrm{LD}}$ & $1.15 \mathrm{Watt} /(\mathrm{Gbit} / \mathrm{s})$ \\
The load independent power $P_{\mathrm{LID}}$ & $5 \mathrm{Watt}$ \\
The power amplifier efficiency of each user $\zeta_{\mathrm{u}, k}$ & 0.2 \\
The power amplifier efficiency at the relay $\zeta_{\mathrm{r}}$ & 0.29 \\
\hline
\end{tabular}

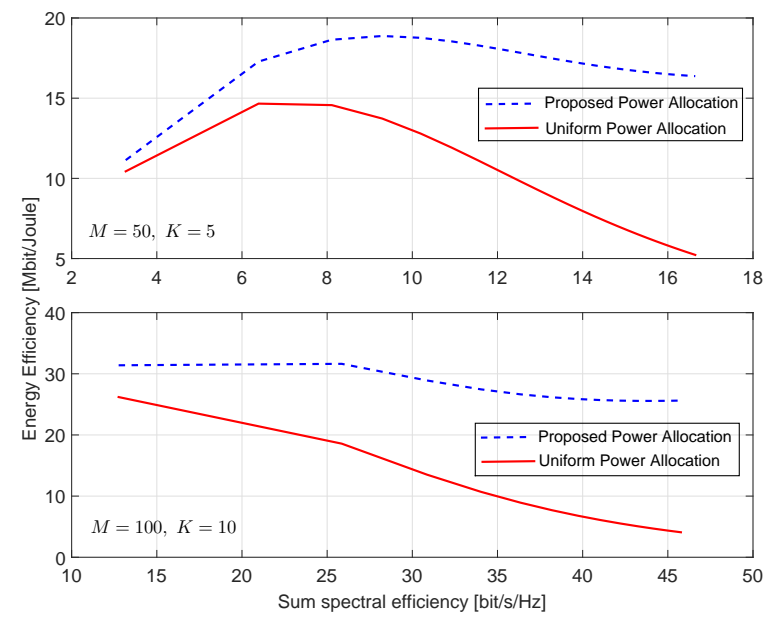

Fig. 5: The energy efficiency versus sum spectral efficiency with the proposed and uniform power allocation schemes for different $M$ and $K$. We choose $P_{\mathrm{r}, \mathrm{th}}=K P_{\mathrm{u}, \mathrm{th}}$ and $\beta_{k}=$ $1, \forall k=1, \ldots, K$.

sum spectral efficiency of the conventional scheme is given by $\mathrm{SE}_{\text {sum }}=\frac{1}{K} \sum_{k=1}^{K} \sum_{t=1}^{K-1} \min \left(\mathrm{R}_{j(k, t)}^{\mathrm{ul}}, \mathrm{R}_{k}^{\mathrm{dl},(t)}\right)$, where $\mathrm{R}_{j(k, t)}^{\mathrm{ul}}$ and $\mathrm{R}_{k}^{\mathrm{dl},(t)}$ are given by (7) and (15), respectively. It can be seen from this figure that the proposed scheme significantly outperforms substantially the conventional transmission scheme. For example, with $K=10, M=200$, the sum spectral efficiency of the proposed scheme is approximately $101 \mathrm{bit} / \mathrm{s} / \mathrm{Hz}$, which is nearly 1.7 times higher than that of the conventional scheme. The reason comes from the fact that our proposed transmission scheme spends only $\left\lceil\frac{K-1}{2}\right\rceil+1$ time-slots for information exchange among the $K$ users, while the conventional transmission scheme spends in total $K$ timeslots. Furthermore, as expected, due to the array gain, the sum spectral efficiencies of both schemes increase when the number of relay antennas increases.

Finally, we examine the benefits of the power allocation algorithms proposed in Sections V and VI. Figure 4 illustrates the comparison of the sum spectral efficiencies without and with power allocation in Section V. For the case of uniform power allocation, we choose $\eta_{j(i, t)}^{(t)}=$ $P_{\mathrm{r}, \mathrm{th}} /\left(M \sum_{i=1}^{K} \beta_{i}\right) \forall i, t, P_{\mathrm{u}, k}=10 \mathrm{~mW}$ for all $k$, and $P_{\mathrm{r}, \mathrm{th}}=100 \mathrm{~mW}$. We can observe that our proposed scheme improves the sum spectral efficiency significantly. For example, at $(M=100, K=10)$ and $(M=200, K=15)$, the proposed power allocation scheme can scale up the spectral efficiency by factors of 1.2 and 1.4, respectively, compared to the case with uniform power allocation. Figure 5 demonstrates the energy
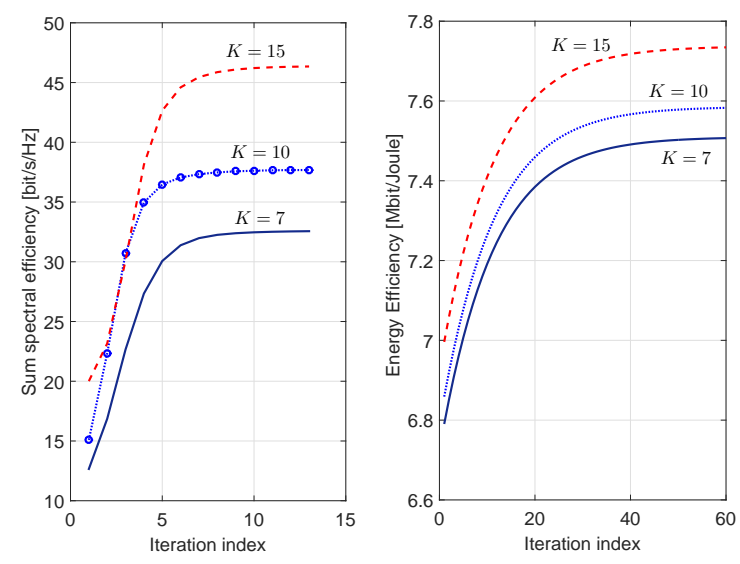

Fig. 6: The convergence of the objective functions in problems (56) and (62) versus the number of iterations. We choose $P_{\mathrm{u}}=$ 0.01 (Watt), $P_{\mathrm{r}}=0.1$ (Watt) for the sum spectral efficiency; and $P_{\mathrm{u}}=0.01$ (Watt), $P_{\mathrm{r}}=K P_{\mathrm{u}}$ (Watt) for energy efficiency.

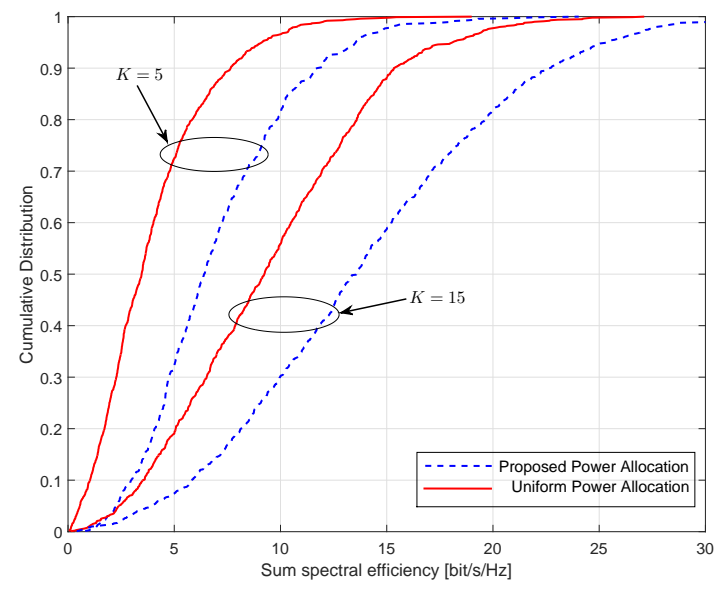

Fig. 7: The cumulative distribution of the sum spectral efficiency with our proposed and uniform power allocation schemes for different $K$. We choose $M=100$.

efficiency versus the sum spectral efficiency, without and with power allocation in Section VI. The parameters used for the simulations are shown in Table I. The proposed power control method provides considerably better system performance than the uniform power allocation method in terms of the energy efficiency. In order to obtain the same sum spectral efficiency of $15 \mathrm{bit} / \mathrm{s} / \mathrm{Hz}$, our proposed scheme can offer a higher energy efficiency by factors of 2.4 and 1.3 for $(M=50, K=5)$ and $(M=100, K=10)$, respectively, compared to uniform power allocation. Moreover, the figure also depicts that in the high power regime, the proposed scheme is more beneficial, i.e., when the sum spectral efficiency increases, the gaps between of two schemes increase.

\section{B. Scenario II}

In this section, we consider a more practical scenario where the large-scale fading $\beta_{k}$ changes depending on the locations 


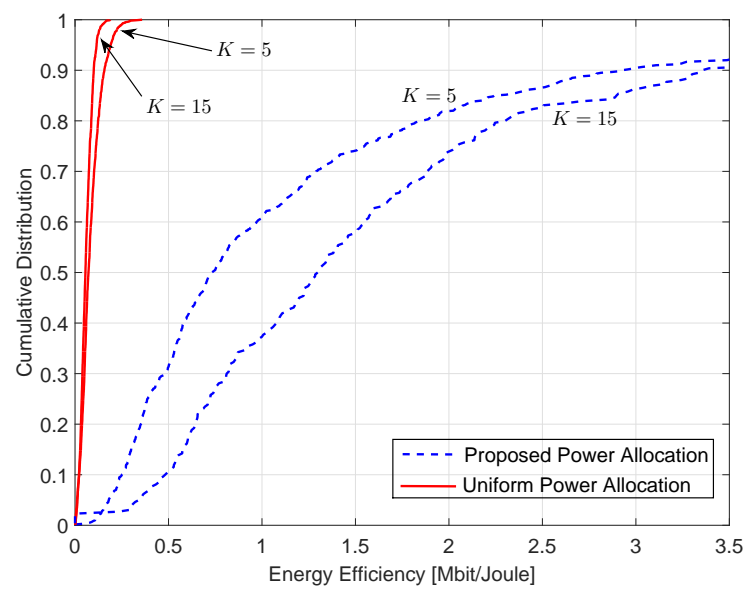

Fig. 8: The cumulative distribution of the energy efficiency, with the proposed and uniform power allocation schemes for different $K$. We choose $M=100$.

of users. In order to generate the large-scale fading, we use the same model as in [12].

First, we examine the convergence of the objective functions in problems (56) and (62). Figure 6 shows the spectral efficiency and energy efficiency versus the number of iterations, for an arbitrary large-scale fading realization. We can see that, Algorithms 1, 2, and 3 converge very fast. Compared to Algorithms 1 and 2, the convergence of Algorithms 3 is slower. The reason comes from the fact that Algorithm 3 is more complicated with a complicated objective function and many constraints.

Next, we compare the performance of our proposed power allocation in Section $\mathrm{V}$ with the performance of uniform power allocation. Figure 7 illustrates the cumulative distribution of the sum spectral efficiency for $M=100$ and different $K$. The dashed curves correspond to the case of our proposed power allocation in Section V, i.e., using Algorithm 1 and Algorithm 2. The solid curves correspond to the case of uniform power allocation, i.e., users and the relay use their full powers: $P_{\mathrm{u}, k}=10 \mathrm{~mW}$ for all $k, P_{\mathrm{r}, \text { th }}=100 \mathrm{~mW}$, and $\eta_{j(i, t)}^{(t)}=P_{\mathrm{r}, \mathrm{th}} /\left(M \sum_{i=1}^{K} \beta_{i}\right)$ for all $i$ and $t$. As can be observed from the figure, the proposed power allocation scheme provides consistently much more sum spectral efficiency compared to that of no power control. Specifically, the proposed power allocation scheme offers $95 \%$-likely sum spectral efficiency approximately 5 and 2 times higher than the uniform power allocation scheme does for $K=5$, and 15 , respectively.

Finally, we evaluate the benefit of the power allocation in Section VI which maximizes the energy efficiency taking into account the effect of power consumption. Again, the parameters used for the simulations are shown in Table I. Figure 8 illustrates the cumulative distribution of the energy efficiency with our proposed and uniform power allocation schemes for $K=5$ and 15. In this example, for the proposed power allocation, we use Algorithm 3, while for the uniform power allocation, the users and the relay use their full powers. Note that, the initial values of Algorithm 3 are chosen as follows: $\epsilon=10^{-2}, \omega=1.1, L=5$, and $\gamma_{k, t}^{(1)}=\min \left(\frac{e_{k, t} P_{\mathrm{u}, \mathrm{h}}}{P_{\mathrm{u}, \mathrm{th}} \sum_{\substack{i=1 \\ i \neq k}}^{K} \beta_{i}+1}, \gamma_{k, \mathrm{th}}^{\mathrm{dl},(t)}\right)$, where $\gamma_{k, \mathrm{th}}^{\mathrm{dl},(t)}$ is given by (66). The choice of $\gamma_{k, t}^{(1)}$ corresponds to the case of uniform power allocation. We can see that, the energy efficiency of our proposed power control method significantly outperforms the uniform power allocation method. It is interesting that with uniform power allocation, the energy efficiency for $K=5$ is higher than that for $K=15$. But this behaviour changes if our proposed power allocation is deployed, i.e., the energy efficiency for $K=5$ is lower than that for $K=15$. This comes from the fact that without power control, full power is used, and hence, by using more users, the power consumption may be very large. As a consequence, the total energy efficiency reduces. By contrast, our proposed scheme optimizes the use of power at each user and at the relay, and hence, the total energy efficiency does increase when $K$ increases.

\section{CONCLUSiOn}

This paper considered a multi-way relay system with DF relaying protocol. In addition, the relay station was equipped with many antennas. We proposed a novel transmission protocol which leverages the massive MIMO technology together with successive cancelation decoding. While the conventional transmission scheme required $K$ time-slots to exchange all information among the $K$ users, our proposed scheme required only $\left\lceil\frac{K-1}{2}\right\rceil+1$ time-slots. As a result, compared to the conventional scheme, our proposed transmission protocol improved significantly the system performance. We also derived an analytical approximation of the sum spectral efficiency. This approximation is very tight, especially when the number of relay antennas is large. Based on this closed-formed expression, we proposed two power allocation algorithms which choose the transmit power at the users and the relay station to maximize the sum spectral efficiency and the energy efficiency. With our proposed power allocation schemes, the system performance (in terms of sum spectral efficiency and energy efficiency) can be significantly improved.

\section{APPENDIX}

\section{A. Proof of Proposition 3}

From (35), the $(m, n)$-th element of $\left(\overline{\mathbf{A}}_{k}^{\left(t^{\prime}\right)}\right)^{H} \overline{\mathbf{A}}_{k}^{\left(t^{\prime}\right)}$ is given by

$$
\begin{aligned}
& {\left[\left(\overline{\mathbf{A}}_{k}^{\left(t^{\prime}\right)}\right)^{H} \overline{\mathbf{A}}_{k}^{\left(t^{\prime}\right)}\right]_{m n}=\sum_{i=1}^{t^{\prime}} \sqrt{\eta_{j\left(k, t^{\prime}+n\right)}^{\left(t^{\prime}-i+1\right)} \eta_{j\left(k, t^{\prime}+m\right)}^{\left(t^{\prime}-i+1\right)}}} \\
& \times \mathbf{g}_{k}^{H} \mathbf{g}_{j(k, i-1+m)} \mathbf{g}_{j(k, i-1+n)}^{H} \mathbf{g}_{k} .
\end{aligned}
$$

Using the trace lemma [20, Lemmas 4, 5], we have

$$
\frac{1}{M} \mathbf{g}_{k}^{H} \mathbf{g}_{j(k, i)} \mathbf{g}_{j(k, i)}^{H} \mathbf{g}_{k}-\frac{\beta_{k}}{M} \operatorname{Tr}\left(\mathbf{g}_{j(k, i)} \mathbf{g}_{j(k, i)}^{H}\right) \underset{M \rightarrow \infty}{\stackrel{a . s .}{\longrightarrow}} 0,
$$

Since $\operatorname{Tr}\left(\mathbf{g}_{j(k, i)} \mathbf{g}_{j(k, i)}^{H}\right)=\left\|\mathbf{g}_{j(k, i)}\right\|^{2}$, and from the law of large numbers, we get

$$
\frac{1}{M} \operatorname{Tr}\left(\mathbf{g}_{j(k, i)} \mathbf{g}_{j(k, i)}^{H}\right) \underset{M \rightarrow \infty}{\stackrel{a . s .}{\longrightarrow}} \beta_{j(k, i)} .
$$


The substitution of (91) into (90) yields

$$
\frac{1}{M} \mathbf{g}_{k}^{H} \mathbf{g}_{j(k, i)} \mathbf{g}_{j(k, i)}^{H} \mathbf{g}_{k} \underset{M \rightarrow \infty}{\stackrel{a . s .}{\longrightarrow}} \beta_{k} \beta_{j(k, i)} .
$$

Similarly, if $i \neq i^{\prime}$, we obtain

$$
\frac{1}{M} \mathbf{g}_{k}^{H} \mathbf{g}_{j(k, i)} \mathbf{g}_{j\left(k, i^{\prime}\right)}^{H} \mathbf{g}_{k} \underset{M \rightarrow \infty}{\stackrel{a . s .}{\longrightarrow}} 0 .
$$

From (89), (92), and (93), we have that the $(n, n)$ th diagonal element of $\frac{1}{M}\left(\overline{\mathbf{A}}_{k}^{\left(t^{\prime}\right)}\right)^{H} \overline{\mathbf{A}}_{k}^{\left(t^{\prime}\right)}$ converges to $\beta_{k} \sum_{i=1}^{t^{\prime}} \eta_{j\left(k, t^{\prime}+n\right)}^{\left(t^{\prime}-i+1\right)} \beta_{j(k, n+i-1)}$, while its off-diagonal elements converge to zero, as $M$ goes to infinity. Therefore, $\frac{1}{M}\left(\overline{\mathbf{A}}_{k}^{\left(t^{\prime}\right)}\right)^{H} \overline{\mathbf{A}}_{k}^{\left(t^{\prime}\right)}$ converges to a diagonal matrix and its inverse converges also to a diagonal matrix whose $(n, n)$-th element is

$$
\begin{aligned}
& {\left[\left(\frac{1}{M}\left(\overline{\mathbf{A}}_{k}^{\left(t^{\prime}\right)}\right)^{H} \overline{\mathbf{A}}_{k}^{\left(t^{\prime}\right)}\right)^{-1}\right]_{n n}} \\
& \stackrel{\text { a.s. }}{\longrightarrow} \frac{1}{\beta_{k} \sum_{i=1}^{t^{\prime}} \eta_{j\left(k, t^{\prime}+n\right)}^{\left(t^{\prime}-i+1\right)} \beta_{j(k, n+i-1)}} .
\end{aligned}
$$

Substituting (94) into (43), we obtain (44).

\section{REFERENCES}

[1] C. D. Ho, H. Q. Ngo, M. Matthaiou, and L. D. Nguyen, "How to scale up the spectral efficiency of multi-way massive MIMO relaying?" in IEEE International Conf. Commun. (ICC), 2018, to appear.

[2] A. Amah and A. Klein, "Non-regenerative multi-way relaying with linear beamforming." in Proc. IEEE PIMRC, Sep. 2009, pp. 1843-1847.

[3] D. Gündüz, A. Yener, A. Goldsmith, and H. V. Poor, "The multiway relay channel," IEEE Trans. Inf. Theory, vol. 59, no. 1, pp. 51-63, Jan. 2013.

[4] Y. Tian and A. Yener, "Degrees of freedom for the MIMO multi-way relay channel," IEEE Trans. Inf. Theory, vol. 60, no. 5, pp. 2495-2511, May 2014.

[5] H. Q. Ngo, M. Matthaiou, and E. G. Larsson, "Massive MIMO with optimal power and training duration allocation," IEEE Wireless Commun. Lett., vol. 3, no. 6, pp. 605-608, Sep. 2014.

[6] T. L. Marzetta, "Massive MIMO: An introduction," Bell Labs Technical Journal, vol. 20, pp. 11-22, 2015.

[7] E. G. Larsson, O. Edfors, F. Tufvesson, and T. L. Marzetta, "Massive MIMO for next generation wireless systems," IEEE Commun. Mag. vol. 52, no. 2, pp. 186-195, Feb. 2014.

[8] E. Björnson, E. G. Larsson, and T. L. Marzetta, "Massive MIMO: Ten myths and one critical question," IEEE Commun. Mag., vol. 54, no. 2, pp. 114-123, Feb. 2016.

[9] J. Hoydis, S. Ten Brink, and M. Debbah, "Massive MIMO in the UL/DL of cellular networks: How many antennas do we need?" IEEE J. Sel. Areas Commun., vol. 31, no. 2, pp. 160-171, Jan. 2013.

[10] H. Yang and T. L. Marzetta, "Performance of conjugate and zeroforcing beamforming in large-scale antenna systems," IEEE J. Sel. Areas Commun., vol. 31, no. 2, pp. 172-179, Jan. 2013.

[11] T. L. Marzetta, E. G. Larsson, H. Yang, and H. Q. Ngo, Fundamentals of Massive MIMO. Cambridge University Press, 2016.

[12] C. D. Ho, H. Q. Ngo, M. Matthaiou, and T. Q. Duong, "On the performance of zero-forcing processing in multi-way massive mimo relay networks," IEEE Commun. Letters, vol. 21, no. 4, pp. 849-852, Apr. 2017

[13] G. Amarasuriya, E. G. Larsson, and H. V. Poor, "Wireless information and power transfer in multi-way massive MIMO relay networks," IEEE Trans. Wireless Commun., vol. 15, no. 6, pp. 3837-3855, June 2015.

[14] G. Amarasuriya and H. V. Poor, "Multi-way amplify-and-forward relay networks with massive MIMO," in Proc. IEEE PIMRC, Sep. 2014, pp. 595-600.

[15] G. Amarasuriya, C. Tellambura, and M. Ardakani, "Multi-way MIMO amplify-and-forward relay networks with zero-forcing transmission," IEEE Trans. Wireless Commun., vol. 61, no. 12, pp. 4847-4863, Dec. 2013.

[16] D. Kudathanthirige and G. A. Baduge, "Multi-cell multi-way massive MIMO relay networks," IEEE Trans. Veh. Technol., 2017.
[17] H. Q. Ngo, E. G. Larsson, and T. L. Marzetta, "Massive MU-MIMO downlink TDD systems with linear precoding and downlink pilots," in Proc. Allerton, Oct. 2013, pp. 293-298.

[18] - "Energy and spectral efficiency of very large multiuser MIMO systems," IEEE Trans. Commun., vol. 61, no. 4, pp. 1436-1449, Apr 2013.

[19] A. M. Tulino and S. Verdú, "Random matrix theory and wireless communications," Foundations and Trends in Commun. and Inf. Theory, vol. 1, no. 1, pp. 1-182, Jun. 2004.

[20] S. Wagner, R. Couillet, M. Debbah, and D. T. Slock, "Large system analysis of linear precoding in MISO broadcast channels with limited feedback," IEEE Trans. Inf. Theory, vol. 58, no. 7, pp. 4509-4537, July 2012.

[21] A. Beck, A. Ben-Tal, and L. Tetruashvili, "A sequential parametric convex approximation method with applications to nonconvex truss topology design problems," J. Glob. Optim., vol. 47, no. 1, pp. 29-51, May 2010.

[22] V. D. Nguyen, T. Q. Duong, H. D. Tuan, O. S. Shin, and H. V. Poor, "Spectral and energy efficiencies in full-fuplex wireless information and power transfer," IEEE Trans. Commun., vol. 65, no. 5, pp. 2220-2233, Feb. 2017.

[23] L. D. Nguyen, H. D. Tuan, T. Q. Duong, and H. V. Poor, "Multi-cell massive MIMO beamforming in assuring QoS for large numbers of users." [Online]. Available: https://arxiv.org/pdf/1712.03548.pdf.

[24] E. Björnson, L. Sanguinetti, J. Hoydis, and M. Debbah, "Optimal design of energy-efficient multi-user MIMO systems: Is massive MIMO the answer?" IEEE Trans. Wireless Commun., vol. 14, no. 6, pp. 3059-3075, Jun. 2015.

[25] S. Boyd and L. Vandenberghe, Convex Optimization. Cambridge, UK: Cambridge University Press, 2004.

[26] M. Grant and S. Boyd, "A MATLAB toolbox for geometric programming," May 2006. [Online]. Available: http://www.stanford. edu/boyd/ggplab/ggplab.pdf.

[27] P. C. Weeraddana, M. Codreanu, M. Latva-aho, and A. Ephremides, "Resource allocation for cross-layer utility maximization in wireless networks," IEEE Trans Veh. Technol., vol. 60, no. 6, pp. 2790-2809, July 2011.

[28] H. Q. Ngo, H. A. Suraweera, M. Matthaiou, and E. G. Larsson, "Multipair full-duplex relaying with massive arrays and linear processing," IEEE J. Sel. Areas Commun., vol. 32, no. 9, pp. 1721-1737, Sep. 2014.

[29] C. D. Ho, H. Q. Ngo, M. Matthaiou, and T. Q. Duong, "Multi-way massive MIMO with maximum-ratio processing and imperfect CSI," in Proc. European Signal Conf. 25th 2017 (EUSIPCO), Aug. 2017, pp. 1704-1708.

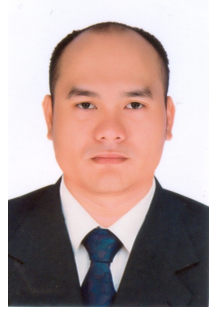

Chung Duc Ho received the B.S. degree in Physics, and M.S. degree in Physical Electronics from Vietnam National University - Ho Chi Minh City University of Natural Sciences, in 2005, and 2010, respectively. He is currently a student research in Electrical, Electronics Engineering and Computer Science at Queen's University Belfast, United Kingdom. His major research interests include MU-MIMO, massive MIMO, and multi-way relay networks with massive MIMO. 


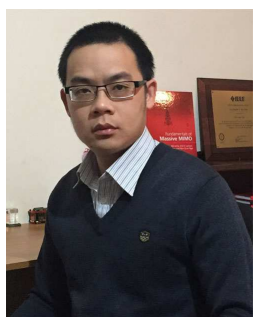

Hien Quoc Ngo received the B.S. degree in electrical engineering from the Ho Chi Minh City University of Technology, Vietnam, in 2007, the M.S. degree in electronics and radio engineering from Kyung Hee University, South Korea, in 2010, and the Ph.D. degree in communication systems from Linköping University (LiU), Sweden, in 2015. In 2014, he visited the Nokia Bell Labs, Murray Hill, New Jersey, USA. From January 2016 to April 2017, Hien Quoc Ngo was a VR researcher at the Department of Electrical Engineering (ISY), LiU. He was also a Visiting Research Fellow at the School of Electronics, Electrical Engineering and Computer Science, Queen's University Belfast, UK, funded by the Swedish Research Council.

Hien Quoc Ngo is currently a Lecturer at Queen's University Belfast, UK. His main research interests include massive (large-scale) MIMO systems, cellfree massive MIMO, physical layer security, and cooperative communications. He has co-authored many research papers in wireless communications and coauthored the Cambridge University Press textbook Fundamentals of Massive MIMO (2016).

Dr. Hien Quoc Ngo received the IEEE ComSoc Stephen O. Rice Prize in Communications Theory in 2015 and the IEEE ComSoc Leonard G. Abraham Prize in 2017. He also received the IEEE Sweden VT-COM-IT Joint Chapter Best Student Journal Paper Award in 2015. He was an IEEE Communications Letters exemplary reviewer for 2014, an IEEE Transactions on Communications exemplary reviewer for 2015, and an IEEE Wireless Communications Letters exemplary reviewer for 2016. Dr. Hien Quoc Ngo currently serves as an Editor for the IEEE Wireless Communications Letters, Digital Signal Processing, and REV Journal on Electronics and Communications. He was a Guest Editor of IET Communications, special issue on "Recent Advances on 5G Communications" and a Guest Editor of IEEE Access, special issue on "Modelling, Analysis, and Design of 5G Ultra-Dense Networks", in 2017. He has been a member of Technical Program Committees for several IEEE conferences such as ICC, Globecom, WCNC, VTC, WCSP, ISWCS, ATC, ComManTel.

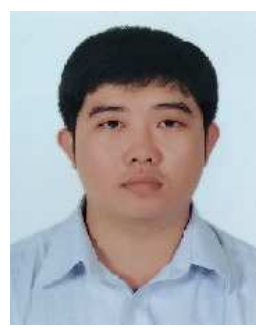

Long D. Nguyen was born in Dongnai, Vietnam. He received the B.S. degree in electronics and telecommunication engineering and the M.S. degree in telecommunication engineering from Ho Chi Minh City University of Technology (HCMUT), Vietnam, in 2013 and 2015, respectively. $\mathrm{He}$ is currently with Queens University Belfast, as a PhD student. His research interests include convex optimization techniques, heterogeneous network, relay networks, and massive MIMO.

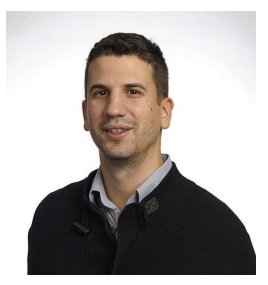

Michail Matthaiou (S'05-M'08-SM'13) was born in Thessaloniki, Greece in 1981. He obtained the Diploma degree (5 years) in Electrical and Computer Engineering from the Aristotle University of Thessaloniki, Greece in 2004. He then received the M.Sc. (with distinction) in Communication Systems and Signal Processing from the University of Bristol, U.K. and Ph.D. degrees from the University of Edinburgh, U.K. in 2005 and 2008, respectively. From September 2008 through May 2010, he was with the Institute for Circuit Theory and Signal Processing, Munich University of Technology (TUM), Germany working as a Postdoctoral Research Associate. He is currently a Reader (equivalent to Associate Professor) in Multiple-Antenna Systems at Queen's University Belfast, U.K. after holding an Assistant Professor position at Chalmers University of Technology, Sweden. His research interests span signal processing for wireless communications, massive MIMO, hardware-constrained communications, and performance analysis of fading channels.

Dr. Matthaiou and his coauthors received the 2017 IEEE Communications Society Leonard G. Abraham Prize. He was the recipient of the 2011 IEEE ComSoc Best Young Researcher Award for the Europe, Middle East and Africa Region and a co-recipient of the 2006 IEEE Communications Chapter Project Prize for the best M.Sc. dissertation in the area of communications. He was co-recipient of the Best Paper Award at the 2014 IEEE International Conference on Communications (ICC) and was an Exemplary Reviewer for IEEE COMMUNICATIONS LETTERS for 2010. In 2014, he received the Research Fund for International Young Scientists from the National Natural Science Foundation of China. In the past, he was an Associate Editor for the IEEE TRANSACTIONS ON COMmUNiCATIONS, Associate Editor/Senior Editor for IEEE COMMUNICATIONS LETTERS and was the Lead Guest Editor of the special issue on "Large-scale multiple antenna wireless systems" of the IEEE Journal on SElected Areas in Communications. He was a co-chair of the Wireless Communications Symposium (WCS) at IEEE GLOBECOM 2016. 